\title{
An annotated checklist of spiders (Arachnida: Aranei) of the National Nature Park 'Buzkyi Hard' (Mykolaiv Area, Ukraine)
}

\author{
Аннотированный список пауков (Arachnida: Aranei) \\ национального природного парка «Бугский ГарА» \\ (Николаевская область, Украина)
}

\author{
N.Yu. Polchaninova ${ }^{1}$, V.A. Gnelitsa ${ }^{2}$, K.V. Evtushenko ${ }^{3}$, \\ E.N. Singaevsky ${ }^{4}$ \\ Н.Ю. Полчанинова ${ }^{1}$, В.А. Гнелица 2 , К.В. Евтушенко \\ Е.Н. Сингаевский ${ }^{4}$
}

\begin{abstract}
${ }^{1}$ V.N. Karazin Kharkiv National University, Maidan Svobody, 4, Kharkiv 61022 Ukraine. E-mail: n.polchaninova@karazin.ua Харьковский национальный университет им. В.Н. Каразина, пл. Свободы, 4, Харьков 61022 Украина

${ }^{2}$ Sumy State University, Rimsky-Korsakov Str. 2, Sumy 40007 Ukraine. E-mail: gnelitsav@gmail.com

Сумской государственный университет, ул. Римского-Корсакова, 2, Сумы 40007 Украина.

${ }^{3}$ I.I. Schmalhausen Institute of Zoology of NAS of Ukraine, B. Khmelnytsky Str., 15, Kyiv 01601 Ukraine. E-mail: evt@izan.kiev.ua Институт зоологии им. И.И. Шмальгаузена Национальной академии наук Украины, ул. Богдана Хмельницкого, 15, Киев 01601 Украина.

${ }^{4}$ Taras Shevchenko National University of Kyiv, Volodymyrska Str., 64/13, Kyiv 01601 Ukraine. E-mail: filantus@gmail.com Киевский национальный университет им. Тараса Шевченко, ул. Владимирская, 64/13, Киев 01601 Украина.
\end{abstract}

KEY WORDS: spiders, European fauna, list of species, conservation areas.

КЛЮЧЕВЫЕ СЛОВА: пауки, фауна Европы, список видов, охраняемые территории.

ABSTRACT. The present annotated checklist of spiders of the National Nature Park 'Buzkyi Hard' (Mykolaiv Area, Ukraine) is based on the author's material collected in 1997, 2006, 2007, 2012, and 2013 . The list includes 266 species of 28 families recorded in the habitats typical of the lower Pivdennyi Buh River region: granite outcrops, forb-bunchgrass steppe, dry and mesic meadows, rocky and floodplain forests, tree plantations, wetlands. It is the first results of long-term arachnological studies in this area. Records of the rare species with mosaic distribution (Neottiura suaveolens, Cyclosa sierrae, Dysdera lata), as well as the species having geographic boundaries in the area at hand (Canariphantes nanus, Mimetus laevigatus, Agyneta saaristoi, Zelotes apricorum), confirm the high conservation value of the National Nature Park 'Buzkyi Hard'.

РЕЗЮМЕ. Данный аннотированный список пауков национального природного парка 'Бугский Гард’ (Николаевская обл., Украина) основан на сборах авторов, проведенных в 1997, 2006, 2007, 2012 и 2013 гг. Список включает 266 видов из 28 ceмейств, собранных в биотопах, типичных для области нижнего течения р. Южный Буг: на гранитных обнажениях, в разнотравно-дерновиннозлаковой степи, на сухих и увлажненных лугах, в наскальных и пойменных лесах, лесопосадках, околовод- ных биотопах. Это первые результаты долговременных арахнологических исследований на данной территории. Находки редких видов с мозаичным распространением (Neottiura suaveolens, Cyclosa sierrae, Dysdera lata), а также видов, имеющих границы ареалов в районе исследования (Canariphantes nanus, Mimetus laevigatus, Agyneta saaristoi, Zelotes apricorum) подтверждают высокую природоохранную ценность НПП 'Бугский Гард’.

\section{Introduction}

A taxonomic inventory is the first step in assessing biodiversity of each locality, and is of particular importance for protected areas. Being a backbone of ecological and geographical studies, species lists provide databases for monitoring fauna and flora changes under protective regimes. Such data can be used in ecological planning and management of nature parks and reserves [Adler, Lauenroth, 2003].

The National Nature Park 'Buzkyi Hard' was established for the conservation of natural landscapes of South Ukraine in 2009. The Park is located at the southwest edge of the Ukrainian Crystalline Shield in the valley of the Pivdennyi Buh River and its two tributaries, Velyka Korabelna and Mertvovod (Fig. 1). According to the geobotanical regioning of Ukraine, the park territory belongs to the steppe zone, the sub- 


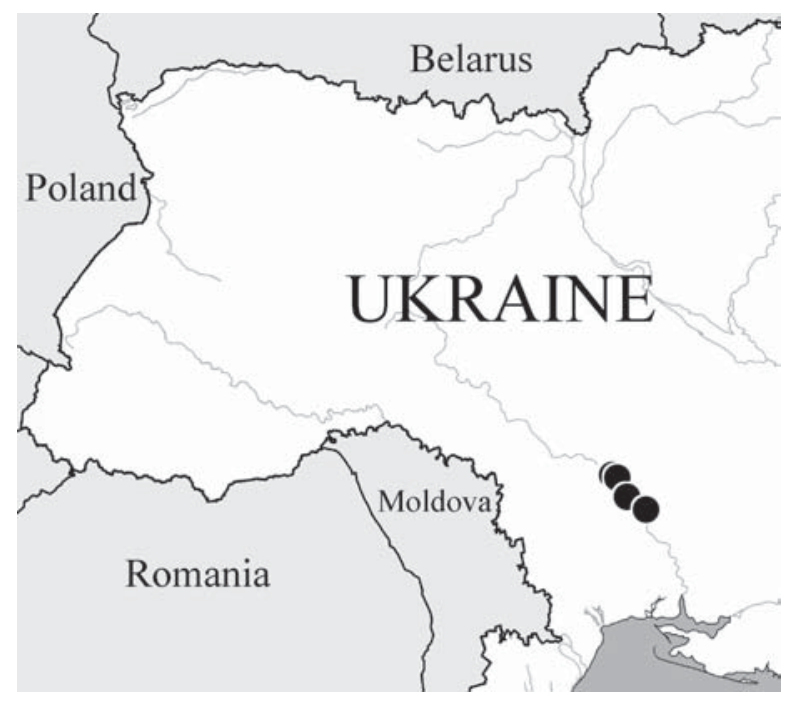

Fig. 1. Map of collecting localities.

Рис. 1. Карта с указанием мест сбора.

zone of northern steppes, Dniester-Buh and Kryvyi Rih districts of forb-bunchgrass steppes, bairak forests (natural forests in the gullies in steppe and forest-steppe zones), and the vegetation of granite outcrops [Barbarych, 1997]. Total area of the Park is 6138.13 ha; it consists of three sectors: Myhiiske, Bohdanivske and Trykratske.

Until now, only nine spider species have been reported for the 'Buzkyi Hard' [Polchaninova, 2015a]. For Alopecosa kovblyuki, the Park represents a westernmost known locality of its range [Nadolny et al., 2012]. Four species recorded from the Park were used as the comparative material in a review of the genus Haplodrassus in the Crimea: H. bohemicus Miller et Buchar, 1977, H. kulczynskii Lohmander, 1942, H. minor (O. Pickard-Cambridge, 1879), and H. pseudosignifer Marusik, Hippa et Koponen, 1996 [Kovblyuk et al., 2012]. Four species were mentioned as rare and patchily distributed in the survey of spiders of Mykolaiv Area: Neottiura suaveolens (Simon, 1879), Cyclosa sierrae Simon, 1870, Mimetus laevigatus (Keyserling, 1863), and Zelotes apricorum (L. Koch, 1876) [Polchaninova, 2015a].

The first arachnological studies along the Pivdennyi Buh River between Myhiya and Yuzhnoukrainsk had been conducted by one of us (K. Evtushenko) in 1997, long before the National Park was established. At that time, the territory had a lower conservation status, simply as a Regional Landscape Park. Then V. Gnelitsa and N.Polchaninova continued investigations in 2006, 2007, 2012 and 2013, and E. Singaevsky added a small collection in 2012 .

The aims of the present paper are (1) to combine all the spider material collected in different years, (2) to compile an annotated list of spiders of the National Nature Park 'Buzkyi Hard' and its vicinity, and (3) to comment on species of a high conservation value.

\section{Material and methods}

Arachnological studies were conducted in June 1997, April-June 2006, May-June 2007, September 2007, April 2012, and August 2013 in the vicinities of the following villages: Kuripchyne $\left(48^{\circ} 00^{\prime} 28^{\prime \prime} \mathrm{N} 31^{\circ} 00^{\prime}\right.$ $\left.57^{\prime \prime} \mathrm{E}\right)$ and Myhiya $\left(48^{\circ} 02^{\prime} 23^{\prime \prime} \mathrm{N} 30^{\circ} 56^{\prime} 48^{\prime \prime} \mathrm{E}\right)$ - the Myhiiske sector of the Park; Bohdanivka $\left(47^{\circ} 48^{\prime} 48^{\prime \prime} \mathrm{N}\right.$ $\left.31^{\circ} 09^{\prime} 23^{\prime \prime} \mathrm{E}\right)$ and Yuzhnoukrainsk $\left(47^{\circ} 49^{\prime} 18^{\prime \prime} \mathrm{N} 31^{\circ}\right.$ $10^{\prime} 30^{\prime \prime} \mathrm{E}$ ) - the Bohdanivske sector of the Park; Aktove $\left(47^{\circ} 42^{\prime} 48^{\prime \prime} \mathrm{N} 31^{\circ} 27^{\prime} 28^{\prime \prime} \mathrm{E}\right)$ and Trykraty $\left(47^{\circ} 42^{\prime} 59^{\prime \prime} \mathrm{N}\right.$ $\left.31^{\circ} 24^{\prime} 17^{\prime \prime} \mathrm{E}\right)$ - the Trykratske sector of the Park.

The material was collected by standard collecting methods:

1. Pitfall trapping. $250 \mathrm{ml}$ plastic caps with the $4 \%$ formalin as preservative were set up in a line of 5-10 traps at a distance of $10 \mathrm{~m}$ from each other in 25 studied habitats: six lines in the Bohdanivske sector (StGr6, W4, F2, F14, FE2, FE6, 4.05-5.06.2007, collector N.Yu. Polchaninova (N.P.); its habitats' description and abbreviations are given in Table 1); 17 lines in the Myhiiske sector (St13, F9, FE4, 3.05-10.06.2006; StGr3, M8, F1, F10, FE1, FE5, 4.05-7.06.2006; StGr1, StGr2, M4, M5, M12, W1, 7.05-9.06.2007, collector N.P.; M10, F12, 22-29.06.1997, collector K.V. Evtushenko - K.E.) and two lines in the Trykraty sector (StGr7, 17-20.04.2012; F22, 18-21.04.2012, collector E.N. Singaevsky - E.S.). A total of 2431 adult spider specimens was collected by pitfall traps.

2. Sweep netting. We used 30-cm diameter entomological sweep-nets and took five repetitions of 25 double-sweeps per habitat in the forb-bunchgrass and granitic steppes, dry, wet and shrub meadows, along the edges of rocky oakeries, flood plain forests and forest plantations. Samples were taken by the corresponding collectors at the same periods as pitfall-trapping (see above), as well as by N.P. on 20.09.2007 and 19-23. 08.2013. This method produced 986 adult individuals.

3 . Hand collecting. The standard procedures of searching spiders under stones, tree bark and stamps, in crevices, etc, the beating of tree brunches and brushing of vertical surfaces produced 552 adult spiders (E.S., K.E., N.P). V.A. Gnelitsa's (V.G.) collection, which was aimed at the Linyphiidae only, resulted in 773 adult specimens.

The total material accounts for 4742 adult spiders recorded from 89 localities.

We arranged collecting localities by the habitat types and provided them with geographic coordinates, name of the nearest village/town, and the collector name (see Table 1).

The recorded taxa are listed below in the alphabetic order following the nomenclature adopted from the World Spider Catalogue [2017] with minor changes. A total number of the collected species in a particular family is given in parenthesis after the family name. We mentioned several species based on juveniles, if adults from a certain Park sector were absent. In the following list, each species is provided with a number 
Table 1. Collecting localities and brief habitat descriptions. Таблица 1. Пункты сборов и краткое описание биотопов.

\begin{tabular}{|c|c|c|c|c|c|}
\hline $\begin{array}{l}\text { Abbre- } \\
\text { viation }\end{array}$ & $\begin{array}{c}\text { Nearest } \\
\text { village/town }\end{array}$ & \multicolumn{2}{|c|}{ Geographic coordinates } & Description & $\begin{array}{c}\text { Collec- } \\
\text { tor }\end{array}$ \\
\hline \multicolumn{6}{|c|}{ ZONAL FORB-BUNCHGRASS STEPPE } \\
\hline St1 & Aktove & $47.7007^{\circ} \mathrm{N}$ & $31.4385^{\circ} \mathrm{E}$ & forb-bunchgrass steppe on the top slopes & NP \\
\hline St2 & Trykraty & $47.7310^{\circ} \mathrm{N}$ & $31.4294^{\circ} \mathrm{E}$ & $\begin{array}{l}5^{\circ}-10^{\circ} \text { E slope, sparse small Crataegus bushes; in } \\
\text { grass }\end{array}$ & VG, ES \\
\hline St3 & Myhiya & $48.0173^{\circ} \mathrm{N}$ & $30.9710^{\circ} \mathrm{E}$ & $\begin{array}{l}\text { flat-interfluves steppe with Cytisus; in grass and } \\
\text { dead plant residues }\end{array}$ & VG \\
\hline St4 & Myhiya & $47.9990^{\circ} \mathrm{N}$ & $31.0021^{\circ} \mathrm{E}$ & $\begin{array}{l}\text { flat-interfluves steppe, sparse Crataegus and } \\
\text { stunted Quercus with grasses, forbs and moss; in } \\
\text { grass and on the soil }\end{array}$ & $\mathrm{VG}$ \\
\hline St5 & Myhiya & $47.9712^{\circ} \mathrm{N}$ & $31.0377^{\circ} \mathrm{E}$ & $\begin{array}{l}\text { steppe by the top hill; in grass near the small } \\
\text { bushes }\end{array}$ & VG \\
\hline St6 & Trykraty & $47.7310^{\circ} \mathrm{N}$ & $31.4294^{\circ} \mathrm{E}$ & flat-interfluves steppe; in grass and on the soil & $\mathrm{VG}$ \\
\hline St7 & Trykraty & $47.7310^{\circ} \mathrm{N}$ & $31.4294^{\circ} \mathrm{E}$ & $\begin{array}{l}\text { narrow gully with steppe vegetation; in grass and } \\
\text { on the soil at the sides of a shallow depression }\end{array}$ & VG \\
\hline St8 & Myhiya & $48.0099^{\circ} \mathrm{N}$ & $30.9788^{\circ} \mathrm{E}$ & $\begin{array}{l}10^{\circ} \mathrm{S} \text { slope along the river, forb-grass steppe with } \\
\text { small bushes }\end{array}$ & $\mathrm{VG}$ \\
\hline St9 & Myhiya & $48.0255^{\circ} \mathrm{N}$ & $30.9627^{\circ} \mathrm{E}$ & $40^{\circ} \mathrm{SW}$ slope of a granite hill; steppe, in grass & VG \\
\hline St10 & Myhiya & $48.0098^{\circ} \mathrm{N}$ & $30.9822^{\circ} \mathrm{E}$ & $\begin{array}{l}20^{\circ} \text { SE slope, steppe with small bushes, } \\
\text { Tragopogon, Thymus, Artemisia; in grass }\end{array}$ & VG \\
\hline St11 & Myhiya & $48.0077^{\circ} \mathrm{N}$ & $30.9865^{\circ} \mathrm{E}$ & $\begin{array}{l}15^{\circ}-20^{\circ} \mathrm{W} \text { slope, gramineous steppe with } \\
\text { Achillea; in grass }\end{array}$ & VG \\
\hline St12 & Trykraty & $47.7310^{\circ} \mathrm{N}$ & $31.4294^{\circ} \mathrm{E}$ & $\begin{array}{l}20^{\circ} \text { E slope, gramineous steppe with small Prunus } \\
\text { bushes; in grass and dead plant residues near the } \\
\text { bushes and in burrows of small rodents }\end{array}$ & VG \\
\hline St13 & Myhiya & $48.0322^{\circ} \mathrm{N}$ & $30.9175^{\circ} \mathrm{E}$ & steppe vegetation on a clayey slope & NP \\
\hline St14 & Myhiya & $48.0186^{\circ} \mathrm{N}$ & $30.9685^{\circ} \mathrm{E}$ & $\begin{array}{l}10^{\circ} \mathrm{S} \text { slope, sparse Crataegus and Rosa, grasses, } \\
\text { forbs; in grass and on the soil }\end{array}$ & VG \\
\hline St15 & Trykraty & $47.7310^{\circ} \mathrm{N}$ & $31.4294^{\circ} \mathrm{E}$ & $\begin{array}{l}\text { E slope, small Crataegus and Prunus bushes with } \\
\text { young Pyrus trees; in grass and in moss }\end{array}$ & $\mathrm{VG}$ \\
\hline \multicolumn{6}{|c|}{ GRANITE OUTCROPS } \\
\hline Gr1 & Yuzhnoukrainsk & $47.8208^{\circ} \mathrm{N}$ & $31.1303^{\circ} \mathrm{E}$ & bare granite outcrops & KE \\
\hline \multicolumn{6}{|c|}{ STEPPE ON THE GRANITIC ROCK (“GRANITIC STEPPE”) } \\
\hline StGr1 & Myhiya & $48.0397^{\circ} \mathrm{N}$ & $30.9713^{\circ} \mathrm{E}$ & granitic steppe & NP \\
\hline StGr2 & Myhiya & $48.0163^{\circ} \mathrm{N}$ & $30.9727^{\circ} \mathrm{E}$ & granitic steppe & NP \\
\hline StGr3 & Kuripchyne & $47.9983^{\circ} \mathrm{N}$ & $30.0022^{\circ} \mathrm{E}$ & granitic steppe & NP \\
\hline StGr4 & Kuripchyne & $47.9978^{\circ} \mathrm{N}$ & $31.0000^{\circ} \mathrm{E}$ & granitic steppe & KE \\
\hline StGr5 & Bohdanivka & $47.8208^{\circ} \mathrm{N}$ & $31.1300^{\circ} \mathrm{E}$ & granitic steppe & KE \\
\hline StGr6 & Yuzhnoukrainsk & $47.8178^{\circ} \mathrm{N}$ & $31.1647^{\circ} \mathrm{E}$ & granitic steppe & NP \\
\hline StGr7 & Myhiya & $48.0171^{\circ} \mathrm{N}$ & $30.9715^{\circ} \mathrm{E}$ & $\begin{array}{l}\text { steppe on a SW slope; in grass and in soil } \\
\text { depressions near the large stones }\end{array}$ & VG, ES \\
\hline StGr8 & Trykraty & $47.7310^{\circ} \mathrm{N}$ & $31.4294^{\circ} \mathrm{E}$ & $\begin{array}{l}5^{\circ}-10^{\circ} \text { E slope, granitic outcrops with the soil } \\
\text { between the stones, moss, lichens, Viola, Iris; in } \\
\text { moss and on the soil }\end{array}$ & VG \\
\hline StGr9 & Trykraty & $47.7310^{\circ} \mathrm{N}$ & $31.4294^{\circ} \mathrm{E}$ & $\begin{array}{l}10^{\circ}-30^{\circ} \text { stony slope, sporadic young Pyrus and } \\
\text { Crataegus, grasses with Ficaria and moss; in grass, } \\
\text { moss and on the soil }\end{array}$ & VG, ES \\
\hline \multicolumn{6}{|c|}{ MEADOWS } \\
\hline M1 & Kuripchyne & $47.8144^{\circ} \mathrm{N}$ & $31.1683^{\circ} \mathrm{E}$ & meadow on the mid slope & $\mathrm{KE}$ \\
\hline M2 & Yuzhnoukrainsk & $47.8217^{\circ} \mathrm{N}$ & $31.1297^{\circ} \mathrm{E}$ & meadow on the mid slope & KE \\
\hline
\end{tabular}


Table 1 (continued). Таблица 1 (продолжение).

\begin{tabular}{|c|c|c|c|c|c|}
\hline $\begin{array}{l}\text { Abbre- } \\
\text { viation }\end{array}$ & $\begin{array}{c}\text { Nearest } \\
\text { village/town }\end{array}$ & \multicolumn{2}{|c|}{ Geographic coordinates } & Description & $\begin{array}{c}\text { Collec- } \\
\text { tor }\end{array}$ \\
\hline \multicolumn{6}{|c|}{ MEADOWS } \\
\hline M3 & Myhiya & $48.0186^{\circ} \mathrm{N}$ & $30.9685^{\circ} \mathrm{E}$ & $\begin{array}{l}25^{\circ} \mathrm{S} \text { slope, sporadic Crataegus with grasses; in } \\
\text { grass, on the soil and in burrows of small rodents }\end{array}$ & VG, ES \\
\hline M4 & Myhiya & $48.0186^{\circ} \mathrm{N}$ & $30.9681^{\circ} \mathrm{E}$ & meadow on the foot slope by the river & NP \\
\hline M5 & Myhiya & $48.0125^{\circ} \mathrm{N}$ & $30.9731^{\circ} \mathrm{E}$ & dry meadow in the river floodplain & NP \\
\hline M6 & Trykraty & $47.7310^{\circ} \mathrm{N}$ & $31.4294^{\circ} \mathrm{E}$ & $\begin{array}{l}\text { rather dry meadow near the river; in grass, on the } \\
\text { soil and in burrows of small rodents }\end{array}$ & VG \\
\hline M7 & Myhiya & $48.0153^{\circ} \mathrm{N}$ & $30.9695^{\circ} \mathrm{E}$ & $\begin{array}{l}10^{\circ} \mathrm{SW} \text { slope of the river bank, meadow, sporadic } \\
\text { Elaeagnus, Crataegus, Sambucus; in grass }\end{array}$ & VG \\
\hline M8 & Kuripchyne & $47.9989^{\circ} \mathrm{N}$ & $30.9942^{\circ} \mathrm{E}$ & shrub meadow in the river floodplain & NP \\
\hline M9 & Myhiya & $47.9950^{\circ} \mathrm{N}$ & $31.0092^{\circ} \mathrm{E}$ & $\begin{array}{l}\text { meadow near the river, sporadic Ulmus, Prunus, } \\
\text { Rosa with moss; in dead leaves near the stones }\end{array}$ & VG, ES \\
\hline M10 & Kuripchyne & $48.0014^{\circ} \mathrm{N}$ & $30.9906^{\circ} \mathrm{E}$ & floodplain meadow & $\mathrm{KE}$ \\
\hline M11 & Yuzhnoukrainsk & $47.8206^{\circ} \mathrm{N}$ & $31.1586^{\circ} \mathrm{E}$ & floodplain meadow & $\mathrm{KE}$ \\
\hline M12 & Myhiya & $48.0164^{\circ} \mathrm{N}$ & $30.9694^{\circ} \mathrm{E}$ & wet meadow in the river floodplain & NP \\
\hline M13 & Myhiya & $48.0267^{\circ} \mathrm{N}$ & $30.9626^{\circ} \mathrm{E}$ & $\begin{array}{l}\text { wet meadow in the river floodplain, solitary Salix } \\
\text { and Amorpha with Calamagrostis, Typha, } \\
\text { Phragmitis, Acorus, Juncus; in grass and in dead } \\
\text { plants near the water }\end{array}$ & $\mathrm{VG}$ \\
\hline \multicolumn{6}{|c|}{ OPEN WOODLANDS } \\
\hline W1 & Myhiya & $48.0392^{\circ} \mathrm{N}$ & $30.9706^{\circ} \mathrm{E}$ & open woodland with shrubs & $\mathrm{NP}$ \\
\hline $\mathbf{W 2}$ & Kuripchyne & $48.0011^{\circ} \mathrm{N}$ & $30.9928^{\circ} \mathrm{E}$ & $\begin{array}{l}\text { sporadic Quercus and Acer trees with shrubs and } \\
\text { grass }\end{array}$ & $\mathrm{KE}$ \\
\hline $\mathbf{W 3}$ & Bohdanivka & $47.8208^{\circ} \mathrm{N}$ & $31.1339^{\circ} \mathrm{E}$ & $\begin{array}{l}\text { sporadic Quercus and Acer trees with shrubs and } \\
\text { grass }\end{array}$ & $\mathrm{KE}$ \\
\hline W4 & Yuzhnoukrainsk & $47.8178^{\circ} \mathrm{N}$ & $31.1639^{\circ} \mathrm{E}$ & open woodland with shrubs & NP \\
\hline W5 & Myhiya & $48.0200^{\circ} \mathrm{N}$ & $30.9697^{\circ} \mathrm{E}$ & $\begin{array}{l}\text { small open pit, young Ulmus, Elaeagnus, } \\
\text { Crataegus with grass; in dead leaves and under the } \\
\text { stones }\end{array}$ & VG \\
\hline W6 & Myhiya & $48.0166^{\circ} \mathrm{N}$ & $30.9701^{\circ} \mathrm{E}$ & $\begin{array}{l}\text { slope near the river, Crataegus brushwood; in } \\
\text { grass }\end{array}$ & VG \\
\hline W7 & Trykraty & $47.7310^{\circ} \mathrm{N}$ & $31.4294^{\circ} \mathrm{E}$ & Crataegus brushwood; in grass & VG \\
\hline \multicolumn{6}{|c|}{ FORESTS } \\
\hline F1 & Kuripchyne & $47.9972^{\circ} \mathrm{N}$ & $30.0117^{\circ} \mathrm{E}$ & $\begin{array}{l}\text { oak forests in the narrow ravines on a steep granitic } \\
\text { river bank, so-called "rocky oakeries" }\end{array}$ & $\mathrm{NP}$ \\
\hline F2 & Yuzhnoukrainsk & $47.8197^{\circ} \mathrm{N}$ & $31.1569^{\circ} \mathrm{E}$ & rocky oakery & NP \\
\hline $\mathbf{F 3}$ & Myhiya & $48.0018^{\circ} \mathrm{N}$ & $30.9909^{\circ} \mathrm{E}$ & $\begin{array}{l}\text { forest on a } 5^{\circ} \mathrm{S} \text { slope, Robinia, Quercus, } \\
\text { Sambucus; in grass and soil litter under fallen } \\
\text { brunches }\end{array}$ & VG \\
\hline F4 & Myhiya & $48.0108^{\circ} \mathrm{N}$ & $30.9824^{\circ} \mathrm{E}$ & $\begin{array}{l}\text { forest on a } 45^{\circ} \text { ESE gully slope, Quercus, Acer; in } \\
\text { dead leaves near the tree trunks }\end{array}$ & VG \\
\hline $\mathbf{F 5}$ & Trykraty & $47.7310^{\circ} \mathrm{N}$ & $31.4294^{\circ} \mathrm{E}$ & $\begin{array}{l}\text { thinness Quercus and Acer with Cornus; in dead } \\
\text { leaves near the stones and tree trunks }\end{array}$ & $\mathrm{VG}$ \\
\hline F6 & Myhiya & $48.0086^{\circ} \mathrm{N}$ & $30.9856^{\circ} \mathrm{E}$ & $\begin{array}{l}\text { deciduous forest in a ravine, Ulmus, Quercus, } \\
\text { Populus with Crataegus and Euonymus; in dead } \\
\text { leaves near the tree trunks }\end{array}$ & VG \\
\hline F7 & Yuzhnoukrainsk & $47.8051^{\circ} \mathrm{N}$ & $31.1686^{\circ} \mathrm{E}$ & deciduous forest in a ravine & $\mathrm{KE}$ \\
\hline F8 & Trykraty & $47.7310^{\circ} \mathrm{N}$ & $31.4294^{\circ} \mathrm{E}$ & $\begin{array}{l}20^{\circ} \mathrm{W} \text { slope, Quercus plantation with Cornus; in } \\
\text { litter and near the tree trunks }\end{array}$ & VG \\
\hline F9 & Myhiya & $48.0314^{\circ} \mathrm{N}$ & $30.9161^{\circ} \mathrm{E}$ & forest plantation in the river floodplain & NP \\
\hline
\end{tabular}


Table 1 (continued). Таблица 1 (продолжение).

\begin{tabular}{|c|c|c|c|c|c|}
\hline $\begin{array}{l}\text { Abbre- } \\
\text { viation }\end{array}$ & $\begin{array}{c}\text { Nearest } \\
\text { village/town }\end{array}$ & \multicolumn{2}{|c|}{ Geographic coordinates } & Description & $\begin{array}{l}\text { Collec- } \\
\text { tor }\end{array}$ \\
\hline \multicolumn{6}{|c|}{ FORESTS } \\
\hline F10 & Kuripchyne & $47.9975^{\circ} \mathrm{N}$ & $30.0027^{\circ} \mathrm{E}$ & forest plantation in the river floodplain & NP \\
\hline F11 & Aktove & $47.7007^{\circ} \mathrm{N}$ & $31.4385^{\circ} \mathrm{E}$ & forest plantation on the lake bank & NP \\
\hline F12 & Kuripchyne & $47^{\circ} 59^{\prime} 58^{\prime \prime} \mathrm{N}$ & $30^{\circ} 59^{\prime} 32^{\prime \prime}$ & floodplain forest & KE \\
\hline F13 & Yuzhnoukrainsk & $47.8117^{\circ} \mathrm{N}$ & $31.1764^{\circ} \mathrm{E}$ & floodplain forest & $\mathrm{KE}$ \\
\hline F14 & Yuzhnoukrainsk & $47.8197^{\circ} \mathrm{N}$ & $31.1569^{\circ} \mathrm{E}$ & floodplain forest & NP \\
\hline F15 & Myhiya & $48.0024^{\circ} \mathrm{N}$ & $30.9903^{\circ} \mathrm{E}$ & $\begin{array}{l}\text { forest on the river bank: Acer, Ulmus, Populus } \\
\text { with grasses and ruderal vegetation; in grass and } \\
\text { on the soil }\end{array}$ & VG \\
\hline F16 & Myhiya & $48.0297^{\circ} \mathrm{N}$ & $30.9404^{\circ} \mathrm{E}$ & $\begin{array}{l}\text { forest on the river bank, Populus, Acer, Salix with } \\
\text { Phragmitis and grass; in grass and dead leaves }\end{array}$ & VG \\
\hline F17 & Myhiya & $48.0297^{\circ} \mathrm{N}$ & $30.9404^{\circ} \mathrm{E}$ & $\begin{array}{l}\text { forest on the river bank, Populus, Acer, Salix with } \\
\text { Phragmitis and grass; in grass and on the soil near } \\
\text { the tree trunks }\end{array}$ & VG \\
\hline F18 & Trykraty & $47.7310^{\circ} \mathrm{N}$ & $31.4294^{\circ} \mathrm{E}$ & $\begin{array}{l}\text { forest on the river bank, } 5^{\circ} \mathrm{S} \text { slope, Quercus, Acer, } \\
\text { Cornus; in dead leaves and on the soil }\end{array}$ & VG \\
\hline F19 & Myhiya & $48.0015^{\circ} \mathrm{N}$ & $30.9912^{\circ} \mathrm{E}$ & $\begin{array}{l}\text { forest on the river bank, Populus, Acer, Ulmus with } \\
\text { Padus bushes; in dead leaves and on the soil near } \\
\text { the Populus trunks }\end{array}$ & VG \\
\hline F20 & Myhiya & $47.9701^{\circ} \mathrm{N}$ & $31.0382^{\circ} \mathrm{E}$ & $\begin{array}{l}\text { floodplain, place between two rocks, Quercus, } \\
\text { Sambucus, Euonymus with moss; in moss and dead } \\
\text { leaves }\end{array}$ & VG \\
\hline F21 & Myhiya & $47.9990^{\circ} \mathrm{N}$ & $31.0021^{\circ} \mathrm{E}$ & $\begin{array}{l}\text { forest on the river bank, Populus, Acer, Ulmus with } \\
\text { Padus bushes; in dead leaves and on the soil near } \\
\text { the Populus trunks }\end{array}$ & VG \\
\hline F22 & Trykraty & $47.7298^{\circ} \mathrm{N}$ & $31.4298^{\circ} \mathrm{E}$ & Quercus plantation with Cornus & ES \\
\hline $\mathbf{F 2 3}$ & Trykraty & $47.7310^{\circ} \mathrm{N}$ & $31.4294^{\circ} \mathrm{E}$ & $\begin{array}{l}\text { Quercus, Fraxinus, Acer forest near the rock, in } \\
\text { litter and near the tree trunks }\end{array}$ & VG \\
\hline \multicolumn{6}{|c|}{ FOREST EDGES } \\
\hline FE1 & Kuripchyne & $47.9972^{\circ} \mathrm{N}$ & $30.0117^{\circ} \mathrm{E}$ & edges of rocky oakeries & $\mathrm{NP}$ \\
\hline FE2 & Yuzhnoukrainsk & $47.8197^{\circ} \mathrm{N}$ & $31.1569^{\circ} \mathrm{E}$ & edges of the oakery on a hill top & NP \\
\hline FE3 & Myhiya & $48.0304^{\circ} \mathrm{N}$ & $30.9440^{\circ} \mathrm{E}$ & edge of a rocky oakery & VG \\
\hline FE4 & Myhiya & $48.0314^{\circ} \mathrm{N}$ & $30.9161^{\circ} \mathrm{E}$ & $\begin{array}{l}\text { edges of the forest plantations in the river } \\
\text { floodplain }\end{array}$ & NP \\
\hline FE5 & Kuripchyne & $47.9978^{\circ} \mathrm{N}$ & $30.0025^{\circ} \mathrm{E}$ & $\begin{array}{l}\text { edges of the forest plantations in the river } \\
\text { floodplain }\end{array}$ & NP \\
\hline FE6 & Yuzhnoukrainsk & $47.8200^{\circ} \mathrm{N}$ & $31.1569^{\circ} \mathrm{E}$ & edge of a floodplain forest & NP \\
\hline FE7 & Aktove & $47.7007^{\circ} \mathrm{N}$ & $31.4385^{\circ} \mathrm{E}$ & edge of a forest plantation on the lake bank & NP \\
\hline FE8 & Myhiya & $48.0101^{\circ} \mathrm{N}$ & $30.9810^{\circ} \mathrm{E}$ & $\begin{array}{l}\text { edge of a gully forest: Quercus, Crataegus, } \\
\text { Euonymus, Sambucus; in dead leaves }\end{array}$ & VG \\
\hline \multicolumn{6}{|c|}{ FOREST SPRINGS } \\
\hline S1 & Myhiya & $48.0086^{\circ} \mathrm{N}$ & $30.9856^{\circ} \mathrm{E}$ & $\begin{array}{l}\text { broad-leaved forest in a gully; in grass between the } \\
\text { roots of trees and bushes near the water }\end{array}$ & VG \\
\hline $\mathbf{S 2}$ & Myhiya & $48.0090^{\circ} \mathrm{N}$ & $30.9855^{\circ} \mathrm{E}$ & $\begin{array}{l}45^{\circ} \mathrm{NW} \text { slope, Acer, Euonymus with grasses and } \\
\text { forbs; in grass near the stones by the water }\end{array}$ & VG \\
\hline \multicolumn{6}{|c|}{ RIVER/LAKE BANKS } \\
\hline B1 & Myhiya & $48.0183^{\circ} \mathrm{N}$ & $30.9682^{\circ} \mathrm{E}$ & $\begin{array}{l}\text { in dead plant remnants among the silt covered } \\
\text { grasses near the water }\end{array}$ & VG \\
\hline B2 & Trykraty & $47.7310^{\circ} \mathrm{N}$ & $31.4294^{\circ} \mathrm{E}$ & $\begin{array}{l}\text { in dead plant remnants among Phragmitis and } \\
\text { Carex near the water }\end{array}$ & VG \\
\hline
\end{tabular}


Table 1 (continued). Таблица 1 (продолжение).

\begin{tabular}{|c|c|c|c|c|c|}
\hline $\begin{array}{l}\text { Abbre- } \\
\text { viation }\end{array}$ & $\begin{array}{c}\text { Nearest } \\
\text { village/town }\end{array}$ & \multicolumn{2}{|c|}{ Geographic coordinates } & Description & $\begin{array}{c}\text { Collec- } \\
\text { tor }\end{array}$ \\
\hline \multicolumn{6}{|c|}{ RIVER/LAKE BANKS } \\
\hline B3 & Myhiya & $48.0153^{\circ} \mathrm{N}$ & $30.9695^{\circ} \mathrm{E}$ & by the water, in grass and Carex covered with silt & $\mathrm{VG}$ \\
\hline B4 & Trykraty & $47.7310^{\circ} \mathrm{N}$ & $31.4294^{\circ} \mathrm{E}$ & Carex, Phragmitis; on the soil near the water & VG \\
\hline B5 & Trykraty & $47.7310^{\circ} \mathrm{N}$ & $31.4294^{\circ} \mathrm{E}$ & $\begin{array}{l}\text { Carex, Phragmitis, Typha; in dead plant remnants, } \\
\text { in grass and on the soil }\end{array}$ & VG \\
\hline B6 & Yuzhnoukrainsk & $47.8214^{\circ} \mathrm{N}$ & $31.1575^{\circ} \mathrm{E}$ & river bank, in riparian vegetation & NP \\
\hline B7 & Myhiya & $48.0308^{\circ} \mathrm{N}$ & $30.9061^{\circ} \mathrm{E}$ & bank of a former river-bed, in riparian vegetation & NP \\
\hline B8 & Myhiya & $48.0233^{\circ} \mathrm{N}$ & $30.9611^{\circ} \mathrm{E}$ & sandy river bank, sparse vegetation & NP \\
\hline B9 & Aktove & $47.7007^{\circ} \mathrm{N}$ & $31.4385^{\circ} \mathrm{E}$ & bank of a man-made lake & NP \\
\hline \multicolumn{6}{|c|}{ DWELLINGS AND HOUSEHOLD BUILDINGS } \\
\hline D1 & Myhiya & $48.0300^{\circ} \mathrm{N}$ & $30.9442^{\circ} \mathrm{E}$ & rural house & NP \\
\hline D2 & Kuripchyne & $48.0036^{\circ} \mathrm{N}$ & $31.0197^{\circ} \mathrm{E}$ & rural houses and sheds & $\mathrm{NP}$ \\
\hline
\end{tabular}

of males/females, the locality code (in square brackets, see Table 1) and collecting date(s). Abbreviations of the collectors' names are given only in the case of joint expeditionary work in the same locality. The localities are arranged by the name of the nearest village/town: A. - Aktove, B. - Bohdanivka, K. - Kuripchyne, M. - Myhiya, T. - Trykraty, Y. - Yuzhnoukrainsk.

The bulk of the studied material is deposited in authors' personal collections. Six species (17 specimens) were transferred to the National Arachnological Collection registered in the V.I. Vernadsky Taurida National University (TNU), Simferopol, the Crimea (curator: M.M. Kovblyuk); 36 species (150 specimens) will be deposited in the collection of the Manchester Museum, UK (curator: D.V. Logunov) and 83 species (161 specimens) in the Zoological Museum of the Moscow State University, Russia (curator: K.G. Mikhailov).

To clarify the specificity of the 'Buzkyi Hard' araneofauna, we have compared it with those of five wellstudied nature parks and reserves that contain the same types of habitats (steppes, dry and wet meadows, natural and/or man-planted forests and forest shelterbelts, shores of water bodies). These conservation areas are situated within the steppe zone of the East European Plain in Ukraine and Russia: the Black Sea State Biosphere Reserve (Kherson Area) [Polchanionva, 2012; VG, unpublished data.], the Ukrainian Steppe Nature Reserve (Kamiani Mohyly and Khomutivskyi Steppe departments, Donetsk Area), the National Nature Park 'Sviati Hory' (Donetsk Area), the Luhansk Natural Reserve (Luhansk Area) [updated lists see in Polchaninova, Prokopenko, 2013, 2017], and the Razdorsky Museum-Reserve (Rostov-on-Don Area) [Ponomarev, Tsvetkova, 2003; A.V. Ponomarev pers. comm.].

A comparative analysis has been performed by means of the program PAST [Hammer et al., 2001], using two methods: combining method (the cluster analysis) and separation method (the correspondence analysis with remote trend, DCA). In the cluster analysis, the Unweighted Pair Group Method with Arithmetic mean (UPGMA) was used as a cluster algorithm and the Bray-Curtis dissimilarity index as a distance measure. In the DCA, values of the axes characterizing their contribution to the spread of data were as follows: axis $1=0.28$, axis $2=0.21$, axis $3=0.11$, axis $4=$ 0.16 .

\section{List of species}

Fam. AGELENIDAE (4)

Agelena labyrinthica (Clerck, 1758)

K. $1 \sigma^{7}$ [M10], 29.06.1997.

Allagelena gracilens C.L. Koch, 1841

A. $4 \sigma^{7} \sigma^{7}$ [FE7], 20.08.2013. M. 1 q [W1], 19.08.2013.

Eratigena agrestis (Walckenaer, 1802)

M. 1 O' [W1], 19.08.2013.

Tegenaria lapicidinarum Spassky, 1934

K. $1 \sigma^{T}[\mathrm{~F} 10], 4.05-7.06 .2006$. M. $1 \sigma^{7}$ [W1], 7.05-9.06.2007. Y. $1 \sigma^{7}$ [StGr6], $1 \sigma^{7}$ [W4], $1 \sigma^{7}$ [F2], 4.05-5.06.2007; 4 ○ $^{7}, 3$ क० [F7], 19.06.1997; 1 ठ [FE6], 4.05-5.06.2007.

\section{Fam. ANYPHAENIDAE (1)}

Anyphaena accentuata (Walckenaer, 1802)

A. 1 juv. [FE7], 20.08.2013. K. 1 \% [F1], $1 \sigma^{7}$ [FE1], 7.05.2006; 1 \& [F10], 3.05-10.06.2006. M. 1 $\sigma^{7}, 1$ 9, 9.06.2007; Y. $1 \sigma^{7}[\mathrm{~F} 2], 1 \sigma^{7}[\mathrm{~F} 14], 1 \sigma^{7}$ [FE2], 1 o [FE6], 4.05-5.06.2007.

Fam. ARANEIDAE (22)

Agalenatea redii (Scopoli, 1763)

K. 2 우 [StGr3], 4 우 [M8], $1 \mathrm{O}^{\top}, 1$ \& [FE1], $1 \bigcirc^{\top}, 1$ + [W2], 7.05.2007. М. $4 \bigcirc^{7}, 9$ 우 [St13], $1 \sigma^{7}$ [B7], 
3.05.2006. T. $1 \bigcirc^{7}$ [St2], 20.04.2012, ES. Y. $1 \bigcirc^{7}, 2$ 우 [StGr 6], 3 +P [FE2], 4.05.2007.

Araneus aff. angulatus Clerck, 1758

Y. 1 \% juv. [FE6], 5.06.2007.

Araneus diadematus Clerck, 1758

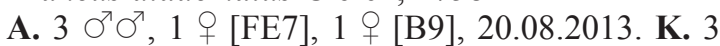
$\sigma^{7} \sigma^{7}, 1$ 우 [FE1], $2 \bigcirc^{7} \sigma^{7}, 5$ 우 [FE5], 22.08.2013. M. 1 $\sigma^{\top}, 2$ 우 [W1], 1 O $^{\top}, 1$ ㅇ [FE4], 1 + [B7], 23.08.2013.

Araneus quadratus Clerck, 1758

A. $1 \mathrm{O}^{7}, 2$ 우 [M14], 20.08.2013. K. 1 우 [M8], 22.08.2013. M. 2 우 [M12], 23.08.2013; 1 ㅇ [F9], 18.09.2006

Araniella cucurbitina (Clerck, 1758)

K. 1 O $^{7}$ [M1], 29.06.1997.

Argiope bruennichi (Scopoli, 1772)

A. 4 우 [M14], 2 우 [B9], 20.08.2013. K. 2 우우 [M8], 4 우 [W2], 1 O , 3 우 [FE1], 1 ㅇ [FE4], 22.08.2013. M. 2 우 [StGr2], 3 웅 [M12], 2 우 [W1], 1 ㅇ [B7], 23.08.2013.

Cercidia prominens (Westring, 1851)

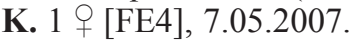

Cyclosa conica (Pallas, 1772)

M. 1 \& [B7], 3.05.2006; K. 1 q [FE1], 7.05.2006; Y. 1 [ [F14], 4.05.2007.

Cyclosa oculata (Walckenaer, 1802)

K. $3 \sigma^{7} \sigma^{7}, 5$ 90 [StGr3], 4 ㅇ [FE1], 7.06.2006. M.

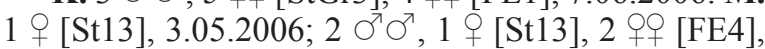
3.06 .2006 .

Cyclosa sierrae Simon, 1870

M. $1 \sigma^{7}$ [W1], 3.06.2006.

Gibbaranea bituberculata (Walckenaer, 1802)

K. $2 \bigcirc^{7} \sigma^{7}, 3$ oᄋ [M8], 07.05.2007; 1 \% [F12], 29.06.1997; $4 \sigma^{7} \sigma^{7}, 6$ 우 [FE1], $1 \sigma^{7}, 4$ oᄋ [FE5],

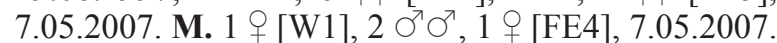
Y. $2 \sigma^{7} \sigma^{\top}$ [StGr6], 1 \% [W2], $2 \sigma^{\top} \sigma^{\top}, 2$ q0 [FE2], 3 oq [FE6], 4.05.2007; 2 ○ $^{\top}$ [F7], 19.06.1997.

Gibbaranea ullrichi (Hahn, 1835)

M. 1 [St13], 9.06.2007.

Hypsosinga pygmaea (Sundevall, 1831)

K. 1 ○ $^{7}$ [StGr3], 7.06.2006.

Larinioides cornutus (Clerck, 1758)

T. $1 \bigcirc^{7}$ [StGr7], 17.04.2012, ES.

Larinioides folium (Schrank, 1803)

A. 1 [ [B9], 20.08.2013. K. 3 + [M8], 4 o [FE1], 7.05.2006. M. $1 \sigma^{7}, 1$ $\left[\right.$ [M12], 20.09.2007. Y. $1 \sigma^{7}, 1$ ㅇ [M11], 19.06.1997; $1 \sigma^{7}, 1+$ [FE2], 1 \% [FE6], 5.06.2007.
Larinioides ixobolus (Thorell, 1873)

M. 2 ㅇ, house wall, 9.06.2007.

Larinioides patagiatus (Clerck, 1758)

K. 2 9 [FE5], 7.05.2006. M. 19 [M5], 7.05.2007;

1 o [FE4], 1 \& [B7], 3.06.2006. Y. 1 [FE6], 4.05.2007.

Mangora acalypha (Walckenaer, 1802)

K. $2 \bigcirc^{7} \bigcirc^{7}, 1$ \& $[\mathrm{StGr} 3], 7.06 .2006 ; 1$ \& [M1], 29.06.1997; 3 ○', 3 क [M8], 7.06.2006; 1 \% [M10], 29.06.1997; $4 \bigcirc^{\top} \sigma^{7}, 2$ OO [FE1], $1 \bigcirc^{7}, 3$ OO [F10], 1 ○", 1 + [FE5], 7.06.2006; M. $2 \sigma^{7} \sigma^{7}$ [St13], 3.05.2006; 5 oo [St13], 1 [FE5], 2 oᄋ [B7] 9.06.2007. Y. 2 oᄋ [StGr6], 4.05.2007; 3 우 [FE2], 5 \%o [FE6], 5.06.2007. A. $1+[\mathrm{M} 14], 1+$ [B9], 20.08.2013.

Neoscona adianta (Walckenaer, 1802)

A. 1 \&FE8], 20.08.2013. M. 1 \% [St13], $1 \sigma^{7}$ [FE4], 3.06.2006. Y. 1 क [StGr5], 29.06.1997; 1 O$^{7}, 1$ + [FE2], 1 [FE6], 5.06.2007.

Singa hamata (Clerck, 1758)

K. 1 \& [M10], 29.06.1997; 1 \& [FE1], 7.06.2006. M. 1 O $^{7}[\mathrm{M} 4], 2 \sigma^{7} \sigma^{7}, 1$ क [M12], 9.06.2007.

Singa nitidula C.L. Koch, 1844

A. 1 \& [B9], 20.08.2013. K. $1 \bigcirc^{7}$ [M1], 29.06.1997;

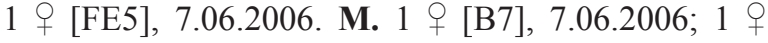
[B7], 9.06.2007.

Zilla diodia (Walckenaer, 1802)

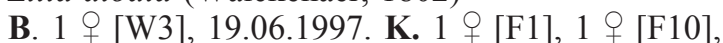
7.05.2006; 2 OO [F10], 7.06.2006; 1 ○', 1 q [FE5],

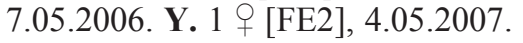

Fam. ATYPIDAE (1)

Atypus muralis Bertkau, 1890

B. $4 \sigma^{7} \sigma^{\top}$ [StGr5], 19.06.1997. K. $1 \sigma^{\top}$ [StGr3], 7.05.2006; 3 ठ $\sigma^{7}$ [StGr4], 29.06.1997; 6 O $^{7} \sigma^{7}$ [M8],

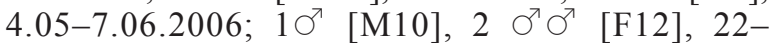
29.06.1997. 3 O $^{7}$ [FE1], 4.05-7.06.2006; M. $10^{7}$ [St13], 3.05-10.06.2006; $1 \sigma^{7}$ [StGr2], $1 \sigma^{7}$ [M4], 7.05-9.06. 2007. T. 1 juv. O [M9], 18.04.2012, ES. Y. $1 \bigcirc^{7}$ [FE6] 4.05-5.06.2007.

\section{Fam. CLUBIONIDAE (5)}

Clubiona caerulescens L. Koch, 1867

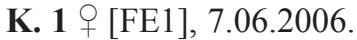

Clubiona lutescens Westring, 1851

K. $2 \sigma^{7} \sigma^{7}[\mathrm{~F} 10], 7.06 .2006$. M. $1 \sigma^{7}, 1$ \% [B7], 7.06.2006. Y. $1 \bigcirc^{7}$ [W4], 05.06.2007.

Clubiona pallidula (Clerck, 1758)

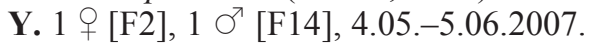

Clubiona phragmitis C.L. Koch, 1843

K. 1 \% [M10], 29.06.1997. 
Clubiona pseudoneglecta Wunderlich, 1994

B. 19 [W3], 19.06.1997. K. $4 \bigcirc^{7} \sigma^{7}[\mathrm{M} 8], 7.06$.

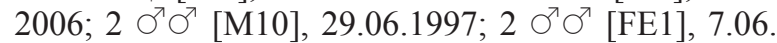

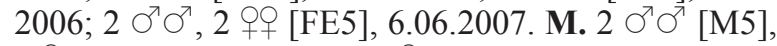
1 q [FE4], 9.06.2007. Y. 1 [FE6], 5.06.2007.

\section{Fam. DICTYNIDAE (6)}

Argenna subnigra (O. Pickard-Cambridge, 1861)

B. 3 우 [W3], 19.06.1997.

Brigittea latens (Fabricus, 1775)

B. 6 ㅇ $[\mathrm{Gr} 1], 19.06 .1997$. K. $5 \sigma^{7} \sigma^{\top}, 7$ ㅇ $[\mathrm{GrSt} 3]$,

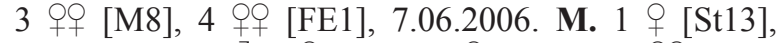
10.06.2006; 1 ○, 1 \% [St13], 1 [ [StGr3], 3 우 [M5],

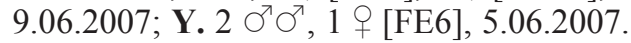

Dictyna arundinacea (Linnaeus, 1758)

A. 1 [FE1], 20.08.2013. K. $6 \bigcirc^{7} \sigma^{7}, 5$ o $[\mathrm{GrSt} 3]$, 5.05.2006; 4 우 [GrSt3], 7.06.2006; 2 ○ ठ [M8], 5.05.2006; [M8], 4 क्ष, 7.06.2006; $5 \sigma^{7} \sigma^{7}, 1$ ㅇ [FE1], 5.05.2006; 4 + [FE1] , 7.06.2006; $2 \sigma^{\top} \sigma^{7}$ [FE4], 5.05.2006; 7 우 [FE4], 7.06.2006. М. $8 \sigma^{7} \sigma^{7}, 20$ Oᄋ

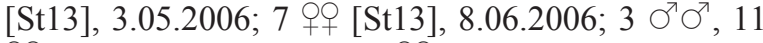
우 [StGr1], 3.05.2006; 2 우 [StGr2], 8.06.2006; 2

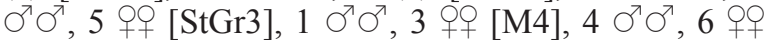
[M5], 7.05.2007; 3 우 [M5], 8.06.2006; $2 \sigma^{7} \sigma^{7}, 2$ of [W1], 7.05.2007; 1 ㅇ [B7], 7.06.2006. Y. $3 \sigma^{\top} \sigma^{\top}, 4$ q0 [StGr6], 4.05.2007; 1 \% [StGr6], 5.06.2007; 2 ठ ठ , 1 + [W4], 2 우 [F2] 4.05.2007.

Dyctina uncinata Thorell, 1856

K. 1 q [FE1], 4.05.2006; 1 क [FE1], 7.06.2006; 1 $\sigma^{7}$ [FE4], 1 ○ [F10], 4.05.2006. M. 1 ㅇ [FE5], 1 ㅇ [B7], 8.06.2006. Y. 1 [W4], 4.05.2007; 2 우 [F13], 19.06.1997; 1 \% [FE1], 4.05.2007.

Lathys stigmatisata (Menge, 1869)

M. $1 \sigma^{7}$ [St13], 3.05-10.06.2006, T. $5 \sigma^{7} \sigma^{7}, 1$ \% [St2], 17-22.04.2012, ES.

Nigma flavescens (Walckenaer, 1830)

A. 1 juv. [FE7], 20.08.2013. K. 2 of [FE5], 4.05.2006. Y. 2 우 [FE2], 04.05.2007.

\section{Fam. DYSDERIDAE (2)}

Dysdera lata Reuss, 1834

B. $1 \sigma^{\top 7}[\mathrm{Gr} 1], 19.06 .1997$.

Harpactea rubicunda (C.L. Koch, 1838)

B. $2 \sigma^{7} \sigma^{7}, 2$ o 2 [W3], 19.06.1997. K. $3 \sigma^{\top} \sigma^{7}, 4$ 우 [W2], 29.06.1997; $1 \sigma^{7}$ [F1], $7 \sigma^{7} \sigma^{7}, 4$ क० [FE1], 4.057.06.2006. M. $2 \sigma^{7} \sigma^{7}$ [StGr1], $1 \sigma^{7}$ [StGr2], $1 \sigma^{7}$ [M4], 7.05-9.06.2007; 3 ठ ठ , 1 우 [W1], 3.05-10.06.2006; 2 ठ $\sigma^{7}$ [W1], 7.05-9.06.2007. T. 1 † [StGr7], 19.04.2012, ES. Y. 10 ㅇ [W4], $3 \sigma^{7} \sigma^{7}, 3$ of [F2], 22

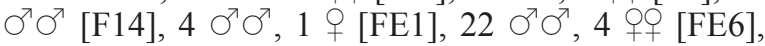
4.05-5.06.2007.

\section{Fam. ERESIDAE (1)}

Eresus kollari Rossi, 1846

M. 1 \& [M3], 21.04.2012, ES.

Fam. EUTRICHURIDAE (5)

Cheiracanthium elegans Thorell, 1875

K. 1 O $^{7}$ [FE5], 7.06.2006; Y. 1 \% [M11], 19.06.1997.

Cheiracanthium mildei L. Koch, 1864

K. 2 우 [StGr3], 7.06.2006; 1 ㅇ [F12], 29.06.1997. 1873

Cheiracanthium pennyi O. Pickard-Cambridge,

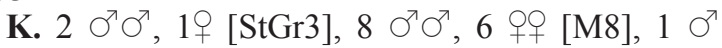
[F12] 29.06.1997; [FE1] $3 \sigma^{7}, 1$ \% , 7.06.2006. M. 1 $\sigma^{7}, 1$ [St13], 8.06.2006; $2 \sigma^{7} \sigma^{7}, 2$ o [St13], $1 \sigma^{7}$ [StGr1], $1 \sigma^{7}$ [M4], 2 ○ $\sigma^{7}, 1$ [ [M5], 9.06.2007. Y. 1

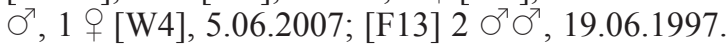

Cheiracanthium. punctorium (Villers, 1789)

M. 1 [M4], 18.09.2006; 10 우 [M12], 22.08.2013.

Cheiracanthium virescens (Sundevall, 1832)

Y. $1 \bigcirc^{7}, 1$ ㅇE6], 5.06.2007.

Fam. GNAPHOSIDAE (26)

Berlandina cinerea (Menge, 1872)

K. $4 \sigma^{7} \sigma^{7}$ [StGr3], $1 \sigma^{7}$ [FE1], 4.05-7.06.2006. M. $5 \sigma^{7} \sigma^{7}$ [StGr1], $2 \sigma^{7} \sigma^{7}, 1$ \% [StGr2], 7.05-9.06. 2007; 4 $\sigma^{7} \sigma^{7}$ [W1], 3.05-10.06.2006. Y. $20 \sigma^{7} \sigma^{7}$ [StGr6], 2 $\sigma^{7} \sigma^{7}$ [FE2], 1 ○', 1 क [FE6], 4.05-5.06.2007.

Civizelotes caucasius (L. Koch, 1866)

B. $3 \sigma^{7} \sigma^{7}, 6$ 우 [Gr1], $4 \sigma^{7} \sigma^{7}, 4$ 우 [StGr5], 19.

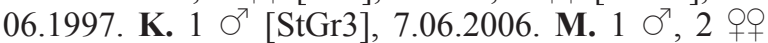
[St13], 7.05.2007; $1 \sigma^{7}$ [StGr2], 7.05-9.06.2007; 1 \% [W1], 9.06.2007.

Civizelotes gracilis (Canestrini, 1868)

B. 4 O $^{7}, 6$ ㅇ [Gr1], 19.06.1997; K. $1 \sigma^{7}$ [M1], 29.06.1997.

Drassodes lapidosus (Walckenaer, 1802)

B. 3 \% [Gr1], $8 \sigma^{7} \sigma^{7}, 15 \%[\mathrm{StGr} 5], 19.06 .1997 . \mathbf{K}$. 1 ○, 1 क [StGr3], 4.05-7.06.2006; 4 ○ $\sigma^{7}$ [M1],

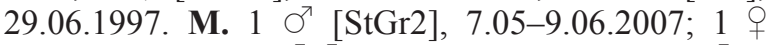

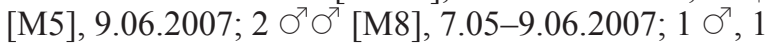
+ [W1], 9.06.2007. Y. $1 \sigma^{7}$ [W4], $1 \sigma^{7}$ [FE2], $1 \sigma^{\top}, 1$ ㅇ [FE6], 4.05-5.06.2007.

Drassodes pubescens (Thorell, 1856)

K. $1 \sigma^{7}$ [FE5], 4.05-7.06.2006. M. $12 \sigma^{7} \sigma^{7}[\mathrm{M} 4], 8$ $\sigma^{7} \sigma^{7}$ [M5], 3 ठ $\sigma^{7}$ [M12], 7.05-9.06.2007.

Drassyllus praeficus (L. Koch, 1866)

K. 3 우 [F12], 22-29.06.1997; 2 O$^{7} \sigma^{7}$ [FE1], 4.057.06.2006. M. $1 \sigma^{7}$ [St13], 3.05-10.06.2006; $1 \sigma^{7}, 1$ q

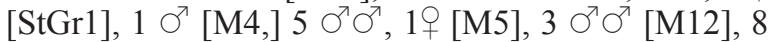
$\sigma^{7}$ [W1], 7.05-9.06.2007; 1 蛋 [FE4], 3.05- 
10.06.2006. Y. $4 \sigma^{7} \sigma^{7}$ [W4], $1 \sigma^{x}$ [F2], $2 \sigma^{7} \sigma^{7}$ [FE2], 5 ठ ठ [FE6], 4.05-5.06.2007.

Drassyllus pumilus (C.L. Koch, 1839)

M. $1 \sigma^{7}$ [St13], (TNU), 3.05-10.06.2006.

Drassyllus pusillus (C.L. Koch, 1833)

K. $1 \Im^{7}$ [M8], $5 \Im^{7} \sigma^{7}$ [FE5], 4.05-7.06.2006; M. 1

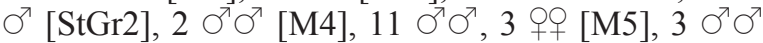
[M12], 7.05-9.06.2007; 1 ○ [W1], 3.05-10.06.2006; $1 \sigma^{7}$ [W1], 7.05-9.06.2007. Y. $1 \sigma^{7}$ [W2], 1 ठ [FE2], $2 \sigma^{7} \sigma^{7}, 1$ 암 [FE6], 4.05-5.06.2007.

Drassyllus vinealis (Kulczyński, 1897)

M. $10^{7}$ [M5], $10^{7}$ [W1], 7.05-9.06.2007.

Gnaphosa taurica Thorell, 1875

B. 3 OO [StGr5], 19.06.1997. K. $1 \sigma^{7}$ [M8], $1 \sigma^{7}$ [F1], 4.05-7.06.2006. М. $2 \sigma^{7} \sigma^{7}$ [M4], 7.05-9.06.2007. Y. $1 \sigma^{\top}, 2$ +o [StGr6], 4 O $^{\top}, 1$ क [W2], $1 \sigma^{\top}$ [F2], 1

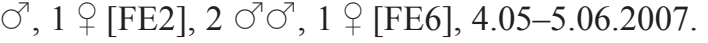

Haplodrassus bohemicus Miller et Buchar, 1977

K. $6 \sigma^{7} \sigma^{7}$, (TNU, Kovblyuk et al., 2012), $2 \sigma^{7} \sigma^{7}, 1$ q [StGr3], 4.05-7.06.2006; M. $5 \sigma^{7} \sigma^{7}$ [St13], $1 \sigma^{7}, 1$ q [StGr1], 3.05-10.06.2006; 10 O $^{\top}, 2$ 우 [StGr2], 7.05-

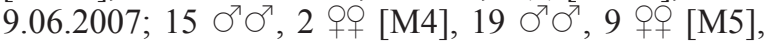
7.05-9.06.2007. Y. $32 \sigma^{7} \sigma^{7}, 6$ oq [StGr6], $3 \sigma^{7} \sigma^{7}$ [W2], $1 \bigcirc^{7}$ [FE6], 4.05-5.06.2007.

Haplodrassus dalmatensis (L. Koch, 1866)

M. $1 \sigma^{7}$ [StGr2], 7.05-9.06.2007.

Haplodrassus kulczynskii Lohmander, 1942

B. 19 [StGr5], 19.06.1997. M. $1 \Im^{7}$, (TNU, Kovblyuk et al., 2012), $2 \sigma^{\top} \sigma^{\top}$ [St13], 3.05-10.06.2006.

Haplodrassus minor (O. Pickard-Cambridge, 1879)

K. $2 \sigma^{\top} \sigma^{\top}$, (TNU, Kovblyuk et al., 2012), $1 \sigma^{7}$ [FE5], 4.05-7.06.2006.

Haplodrassus pseudosignifer Marusik, Hippa et Koponen, 1996

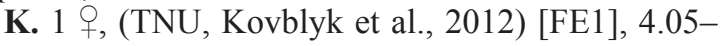
7.06.2006

Haplodrassus. signifer (C.L. Koch, 1839)

B. 1 $[\mathrm{W} 3], 19.06 .1997$. M. $1 \bigcirc^{7}[\mathrm{St13}], 3.05-$ 7.06.2006.

Haplodrassus silvestris (Blackwall, 1833)

Y. 3 우 [F14], 4.05.2007.

Haplodrassus umbratilis (L. Koch, 1866)

Y. $1 \sigma^{7}$ [FE2], 4.05-5.06.2007.

Leptodrassex memorialis (Spassky, 1940)

B. $1 \bigcirc^{\top}, 4$ O+ [Gr1], 19.06.1997.

Micaria dives (Lucas, 1846)

K. $1 O^{7}$ [StGr3], 7.06.2006.
Nomisia aussereri (L. Koch, 1872)

M. 1 \& [StGr2], 23.08.2013.

Nomisia exornata (C.L. Koch, 1839)

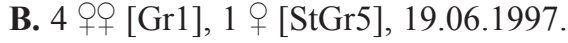

Trachyzelotes pedestris (C.L. Koch, 1837)

K. 1 党 [M1], 29.06.1997; $2 \sigma^{7} \sigma^{7}$ [M8], 4.057.06.2006; 1 ○, 1 \% [F12], 22-29.06.1997. М. $1 \sigma^{7}$ [StGr1], 3.05-10.06.2006; $1 \sigma^{7}$ [M4], $1 \sigma^{7}$ [M12], 2 $\sigma^{7} \sigma^{7}$ [W1], 7.05-9.06.2007; 1 蛋 [FE5], 3.0510.06.2006. Y. $1 \sigma^{\top}[\mathrm{FE} 1], 4 \sigma^{\top}$ [F2], 1 ○, 1 \% [FE6], 1 o [F14], 4.05-5.06.2007.

Zelotes apricorum (L. Koch, 1876)

B. $2 \sigma^{7} \sigma^{7}[\mathrm{Gr} 1], 19.06 .1997$. K. $1 \sigma^{7}[\mathrm{M} 8], 2 \sigma^{7} \sigma^{7}$ (TNU) [F1], 4.05-7.06.2006; 3 ठ $\sigma^{7}$ [F12], 22-29. 06.1997; $1 \bigcirc^{7}$ [FE1], 4.05-7.06.2006. M. 4 +O (TNU), $2 \sigma^{7} \sigma^{7}$ [W1], 3.05-10.06.2006; $1 \sigma^{7}, 1$ \% [W1], 7.059.06.2007. T. $2 \sigma^{7} \sigma^{7}$ [F22], 18-21.04.2012. Y. $1 \sigma^{7}$ [F7], 19.06.1997; 1 ठ [TE2], 4.05-5.06.2007.

Zelotes electus (C.L. Koch, 1839)

K. $1 \sigma^{7}$ [StGr3], 7.05.2006; 4 우 [M8], $3 \sigma^{7} \sigma^{7}, 1$ ㅇ [FE5], 4.05-7.06.2006; М. $7 \sigma^{7} \sigma^{7}, 1$ + [StGr1], 3.0510.06.2006; $1 \sigma^{7}$ [StGr1], 1 \% [StGr2], 7.05-9.06.2007; 13 ○ $^{7}, 2$ 우 [M4], 6 ○ $\sigma^{7}$ [M5], 7.05-9.06.2007; 2 $\sigma^{7} \sigma^{\top}, 2$ 우 [W1], 3.05-10.06.2006; T. $1 \sigma^{\top}$ [St2], 1722.04.2012, ES. Y. 7 ○ $^{7}, 2$ 90 [StGr6], 6 O $^{7} \sigma^{7}$ [W2], $1 \sigma^{7}$ [FE6], 4.05-5.06.2007.

Zelotes longipes (L. Koch, 1866)

Y. $1 \sigma^{7}$ [StGr6], 4.05-5.06.2007; K. 1 [StGr3], 4.05-7.06.2006; $1 \sigma^{7}$ [M1], 29.06.1997. М. $1 \sigma^{7}$ [StGr2], 7.05-9.06.2007; $1 \mathrm{O}^{7}$ [W1], 3.05-10.06.2006; T. 1 ㅇ [St2], 17-22.04.2012, ES.

Fam. HAHNIDAE (1)

Hahnia pusilla C.L. Koch, 1841

T. $1 \sigma^{7}$ [StGr7], 19.04.2012, ES.

Fam. LINYPHIIDAE (61)

Abacoproeces saltuum (L. Koch, 1872)

K. $1 \sigma^{7}$ [F12], 22-29.06.1997. M. $1 \sigma^{7}, 2$ oq [W1], 7.05-9.06.2007. Y. 2 O $^{7}, 1$ क [F14], 1 ค [FE6], 4.055.06.2007. 1872)

Acartauchenius scurrilis (O. Pickard-Cambridge,

T. 2 O $^{7}, 1$ क [StGr9], 18.04.2012, ES; 1 \& [StGr9], 20.04.2012, VG. Y. 4 O+ [F13], 19.06.1997.

Agyneta fuscipalpa (C.L. Koch, 1836)

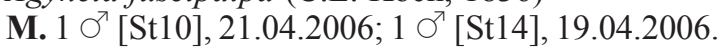
T. $1 \sigma^{\top}$ [StGr9], 20.04.2012, VG.

Agyneta rurestris (C.L. Koch, 1836)

K. $1 \sigma^{7}$ [FE1], 7.05.2006; 1 \% [FE1], 6.06.2007; 1

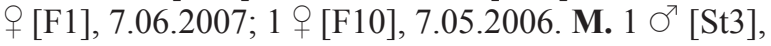


20.04.2006; 1 ○ा, 2 우 [St5], 26.04.2006; 2 ㅇ [St9],

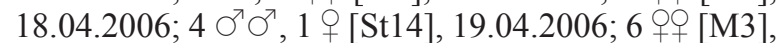
19.04.2006; 1 ठ [W5], 19.04.2006; 1 त [F21], 24.04.2006. T. 1 ㅇ [StGr7], 19.04.2012; 4 우 [StGr7],

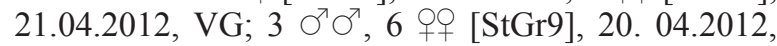
$\mathrm{VG} ; 1$ ㅇ [W7], 18.04.2012.

Agyneta saaristoi Tanasevitch, 2000

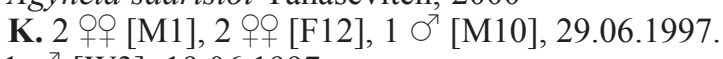
Y. 1 O $^{\top}$ [W3], 19.06.1997.

Agyneta simplicitarsis (Simon, 1884)

K. 2 O$^{\top} \sigma^{7}, 3$ 우 [M1], $1 \sigma^{\top}$ [F12], 29.06.1997. M. 4 $\sigma^{\top} \sigma^{\top}, 1$ [ [St3], 20.04.2006; $5 \sigma^{\top} \sigma^{7}, 2$ o 9 [St4], 24. 04.2006; 1 [ [St8], 22.04.2006; $1 \sigma^{7}$ [St9], 18.04.2006; $1 \sigma^{\top}$ [St11], 22.04.2006; $1 \sigma^{\top}$ [St14], 19.04.2006; 1 ㅇ [M3], 19.04.2006; 2 O $\sigma^{7}$ [M7], 20.04.2006; $1 \sigma^{7}$ [W5], 19.04.2006; 1 ○ [W6], 20.04.2006; 2 우 [F21], 24.04.2006. Y. 1 ㅇ [M2], 19.06.1997.

Araeoncus humilis (Blackwall, 1841)

K. 1 [F1], 7.05.2006. M. 1 \&St3], 20.04.2006; 1 ㅇ $[\mathrm{M} 13], 18.04 .2006$. T. $1 \sigma^{\top}$ [StGr7], 21.04.2012, $\mathrm{VG}$.

Bathyphantes approximatus (O. Pickard-Cambridge, 1871)

M. 1 [M13], 18.04.2006.

Bathyphantes gracilis (Blackwall, 1841)

M. 2 +⿱ [S1], 21.04.2006; $1 \sigma^{\top}$ [St14], 1 \% [M3],

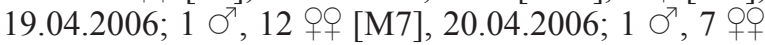
[M13], 18.04.2006; 1 O', 1 ㅇ [F16], 17.04.2006; 3 $\sigma^{\top} \sigma^{\top}, 1$ [B3], 20.04.2006. T. $1 \sigma^{\top}$ [B4], 20.04.2012. Y. $2 \sigma^{\top} \sigma^{\top}, 1$ \% [F13], 19.06.1997.

Bathyphantes nigrinus (Westring, 1851)

K. 1 [FE1], 07.05.2006. M. 1 [F3], 23.04.2006;

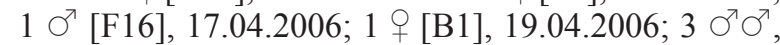
$1+$ [B3], 20.04.2006; $2 \sigma^{\top} \sigma^{\top}, 1$ \& [B7], 03.05.2006.

Canariphantes nanus (Kulczyński, 1898)

M. 1 [StGr7], 20.04.2006, VG; 1 [F20], 26.04. 2006. T. $1+$ [St2], 21.04.2012, VG; $1+$ [St12], 18. 04.2012; 1 ㅇ [F5], 17.04.2012; 1 क [F8], 14.04.2012.

Centromerus capucinus (Simon, 1884)

T. 5 우 [St7], 19.04.2012.

Centromerus sylvaticus (Blackwall, 1841)

K. 1 \& [FE5], 7.05.2006. M. 1 \& [S2], 23.04.2006;

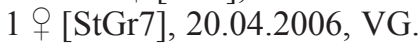

Ceratinella brevis (Wider, 1834)

K. $1 \sigma^{7}[\mathrm{~F} 1], 7.06 .2006 ; 1$ 和10], 3.05.2007. M. 1 $\sigma^{7}$ [St5], 26.04.2006; 1 ऽ, 3 ơ [St14], 19.04.2006; 1 $\sigma^{7}, 3$ 우 [F15], 23.04.2006; 1 ○ [FE8], 21.04.2006. T. 1 o [StGr7], 19.04.2012, ES; 1 [ [M6], 17.04.2012; 1 $\sigma^{7}$ [F5], 17.04.2012. Y. $1 \sigma^{7}$ [F2], 3.05.2007.

Dactilipisthes digiticeps (Simon, 1881)

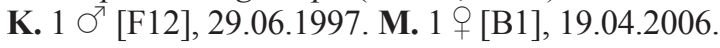

Diplocephalus cristatus (Blackwall, 1833)

M. $3 \sigma^{7} \sigma^{7}, 1$ [F16], 17.04.2006; $1 \sigma^{7}, 1$ [B1],

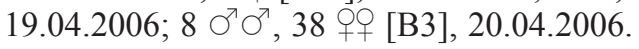

Diplocephalus picinus (Blackwall, 1841)

K. 1 \& [F10], 7.06.2006. M. 1 \& [F17], 17.04.2006.

Diplostyla concolor (Wider, 1834)

K. 1 ठ [M10], 22-29.06.1997. М. 1 ○, 6 우 [M7], 20.04.2006; 2 ๙ $\sigma^{7}, 2$ o+ [M9], 25.04.2006; 1

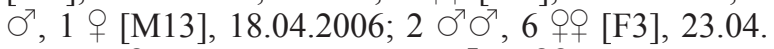
2006; 1 ㅇ [F6], 21.04.2006; 1 ठ , 4 우 [F15], 23.04. 2006; 1 ㅇ [F16], 17.04.2006; 2 우 [F19], 24.04.2006; 1 \% [FE5], 3.05-10.06.2006; 1 ㅇ [B1], 19.04.2006; 1 $\mathrm{O}^{7}, 10$ 우 [B3], 20.04.2006; 1 ○', 2 우 [B7], 3.05.2007; 1 ㅇ [S2], 23.04.2006.

Donacochara speciosa (Thorell, 1875)

M. 2 ㅇ [F16], 17.04.2006.

Erigone dentipalpis (Wider, 1834)

K. 1 \& [F12], 29.06.1997. M. 1 [B7], 3.05.2007. Y. 1 [B6], 04.05.2007.

Gnathonarium dentatum (Wider, 1834)

K. $1 \sigma^{7}$ [F12], 29.06.1997. M. $9 \sigma^{7} \sigma^{7}, 31$ 우 [M13],

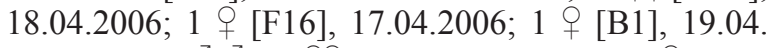
2006; T. $3 \sigma^{7} \sigma^{7}, 3$ O० [B2], 18.04.2012; 1 q [B5], 22.04.2012. Y. 1 ○', 1 \% [FE5], 4.05.2007.

Gongylidiellum murcidum Simon, 1884

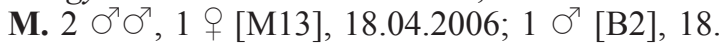
04.2012. T. $1 \sigma^{7}$ [B4], 20.04.2012.

Hypomma cornutum (Blackwall, 1833)

T. 1 [B2], 18.04.2012; 1 [ [B5], 22.04.2012.

Hypsocephalus pusillus (Menge, 1869)

M. 2 + [St11], 22.04.2006.

Ipa keyserlingi (Ausserer, 1867)

M. 1 क [St5], 26.04.2006; 1 + [St11], 22.04.2006.

Lepthyphantes leprosus (Ohlert, 1865)

B. $7 \Im^{7} \sigma^{7}, 3$ ㅇ [W3], 19.06.1997; M. 1 \% [W5], 19.04.2006; 3 oo [M9], 25.04.2006. T. 1 \% [StGr9], 20.04.2012, VG.

Linyphia hortensis Sundevall, 1830

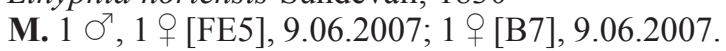

Linyphia triangularis (Clerck, 1758)

A. $1 \sigma^{7}, 13$ 우 [F11], 20.08.2013; $1 \sigma^{\top}, 6$ 우 [FE7], 6 우 [B9], 20.08.2013; K. 1 ㅇ [M8], 3 우 [FE1], 8 우 [FE5], 22.08.2013; 1 † [F12], 29.06.1997. M. 7 oq [F9], 19.08.2013; 2 क् [B7], 20.09.2007; 1 ठ , 4 o० [B7], 22.08.2013.

Mecopisthes peusi Wunderlich, 1972

T. 2 우 [St2], 21.04.2012; 1 [ [St7], 19.04.2012; 15 우 [StGr7], 21.04.2012; 8 우 [StGr9], 20.04.2012, $\mathrm{VG} ; 1$ ㅇ [M6], 17.04.2012. 
Metopobactrus ascitus (Kulczyński, 1894):

M. 2 ㅇ [F17], 17.04.2006.

Micrargus laudatus (O. Pickard-Cambridge, 1881)

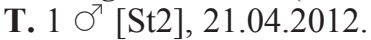

Microlinyphia impigra (O. Pickard-Cambridge, 1871) 2006

M. $1 \bigcirc^{7}, 1$ \& [M13], 18.04.2006; 1 O $^{7}$ [F17], 17.04 .

Microlinyphia pusilla (Sundevall, 1830)

K. 1 [F10], 4 우 [FE1], 1 ○ [FE5], 7.05.2006;

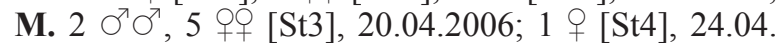
2006; 1 ○’, 2 우 [St8], 22.04.2006; 2 우 [St9], 18.04 2006; 1 ○', 1 [St10], 21.04.2006; 1 + [St11], 22.04. 2006; 1 ○', 2 우 [St14], 1 ○', 2 우 [M3], 19.04.2006; 1 $\sigma^{\top}$ [B7], 3.05.2006; T. $1 \sigma^{\top}, 2$ \%० [StGr7], 21.04.2012.

Microneta viaria (Blackwall, 1841)

K. 1 1 [F1], 7.05.2006; Y. $2 \sigma^{7} \sigma^{7}, 3$ oᄋ [F14], 4.05.2007.

Minicia marginella (Wider, 1834)

T. $1 \sigma^{7}$ [St6], 17.04.2012; $4 \sigma^{7} \sigma^{7}, 4$ oᄋ [St12], 18.04.2012; 6 O $^{\top}, 4$ 우 [St15], 21.04.2012; 2 \%ᄋ [StGr7], 20.04.2012, ES; 1 ○', 1 क [M6], 17.04.2012; 3 $\sigma^{\top} \sigma^{\top}, 3$ 우 [W7], 18.04.2012.

Mioxena blanda (Simon, 1884)

M. 2 우 [F17], 17.04.2006.

Neriene clathrata (Sundevall, 1830)

K. 5 우 [M8], 7.06.2006; 1 O', 1 क [F1], 7.05.2006; 1 + [FE1], 7.05.2006; M. $2 \bigcirc^{7} \sigma^{7}, 2$ 우 [M7], 20.04.

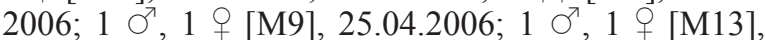
18.04.2006; 1 ○, 1 † [F3], 23.04.2006; 1 [F6], 21.

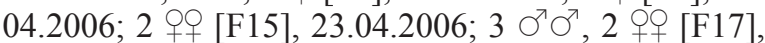
17.04.2006; 1 ㅇ [F19], 24.04.2006; 1 ㅇ [F20],

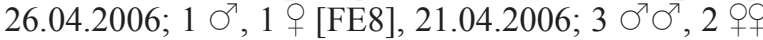
[B3], 20.04.2006; 1 \% [B7], 3.05.2006; 2 우 [B7], 8. 06.2007; 1 [ [S2], 23.04.2006. T. 3 O $^{\top}, 6$ 우 [St15], $21.04 .2012 ; 5$ 우 [W7], 18.04.2012; 2 우 [F5], 17.04.2012; 1 \% , 1 ㅇ [F8], 19.04.2012; 2 우 [F18], 18.04.2012; 1 ㅇ $[\mathrm{F} 23], 20.04 .2012 ; 1$ 우 [B5],

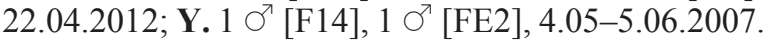

Neriene montana (Clerck, 1758)

M. $1 \sigma^{7}$ [F15], 23.04.2006; 1 丁 $^{7}$ [F17], 17.04.2006.

Neriene radiata (Walckenaer, 1841)

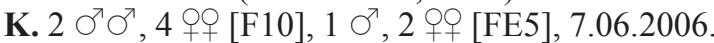
M. 3 우 [S2], 1 O', 4 아 [F3], 23.04.2006. T. 2 우 [F5], 17.04.2012; 2 우 [F8], 19.04.2012; 1 우 [B5], 22.04.2012. Y. 1 ㅇ [F13], 19.06.1997.

Oedothorax apicatus (Blackwall, 1850)

K. $1 \sigma^{\top}$ [F12], 29.06.1997. M. $1 \sigma^{\top}, 4$ 우 [B1], 19.04.2006. Y. 1 i [M11], 19.06.1997.

Oedothorax fuscus (Blackwall, 1834)

K. $3 \sigma^{\top} \sigma^{\top}, 1$ \% [F12], 22-29.06.1997; 1 \% [FE5], 4.05-5.06.2007. M. 1 [FE3], 3.05.2006.
Oedothorax retusus (Westring, 1851)

M. 1 [B7], 3.05.2006.

Panamomops mengei Simon, 1926

T. 1 \% [F23], 20.04.2012. Y. $1 \bigcirc^{\text {T }}$ [FE2], 1 क [FE5], 4.05-5.06.2007.

Pocadicnemis juncea Locket et Millidge, 1953

T. $1 \overbrace{}^{T}$ [B4], 20.04.2012.

Porrhomma microphthalmum (O. Pickard-Cambridge, 1871)

T. $1 \sigma^{T}$ [St6], 17.04.2012.

Porrhomma pygmaeum (Blackwall, 1834)

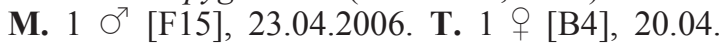
2012; 1 ㅇ [B5], 22.04.2012.

Silometopus reussi (Thorell, 1871)

Y. 1 \& [F13], 19.06.1997.

Stemonyphantes lineatus (Linnaeus, 1758)

M. 1 o $[\mathrm{St} 10], 21.04 .2006 ; 1$ \% [StGr2], 4.055.06.2007; 2 ㅇ [StGr7], 20.04.2006; $1 \sigma^{7}, 1$ \% [M3], 19.04.2006; $1 \sigma^{\text {T }, ~} 1$ ㅇ [M5], 4.05-5.06.2007; 1 \% [B3], 20.04.2006.

Tapinocyboides pygmaeus (Menge, 1869)

Y. 1 O [F13], 19.06.1997.

Tenuiphantes flavipes (Blackwall, 1854)

A. $1 \sigma^{7}$ [B9], 20.08.2013. B. 7 우 [W3], 19.06 . 1997; K. 2 OO [F12], 29.06.1997. М. 5 O$^{7} \sigma^{7}, 1$ क [M9], 25.04.2006, VG; $2 \sigma^{7} \sigma^{7}, 11$ OQ [F3], 23.04.2006; 4

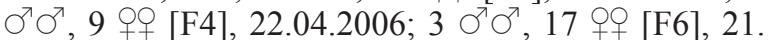
04.2006; $1 \sigma^{\top}$ [F17], 17.04.2006; $2 \sigma^{7} \sigma^{7}, 8$ + 8 [F19],

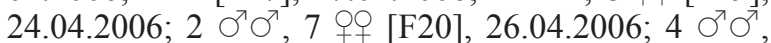
10 우 [FE8], 21.04.2006; 1 o [B7], 7.06.2006; 1 ㅇ

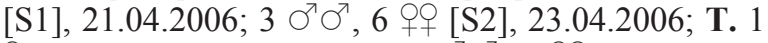
O [StGr7], 21.04.2012, VG; 5 O $^{7}, 4$ 우 [F5], 17.04.

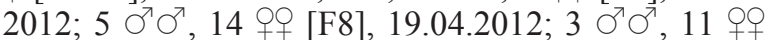
[F18], 18.04.2012; $3 \sigma^{7} \sigma^{7}$ [F22], 18-21.04.2012; 4 $\sigma^{\top} \sigma^{\top}, 10$ कᄋ [F23], 20.04.2012. Y. 1 O $^{\Upsilon}$ [F2], 4. 05.2007.

Tenuiphantes tenuis (Blackwall, 1852)

M. 1 + [M9], 25.04.2006, VG.

Trichoncoides piscator (Simon, 1884)

T. 1 i [St2], 21.04.2012.

Trichoncus hackmani Millidge, 1955

K. 1 [FE1], 7.06.2006. М. $2 \sigma^{\top} \sigma^{7}, 5$ ㅇ [St3], 20.04.2006. T. 2 + 9 [St7], 19.04.2012.

Trichopterna cito (O. Pickard-Cambridge, 1872)

K. 2 ㅇ [M8], 4.05-7.06.2006. M. $1 \sigma^{7}, 3$ ㅇ [St3], 20.04.2006; 7 우 [St4], 24.04.2006; 4 Oे $^{7}, 11$ 우

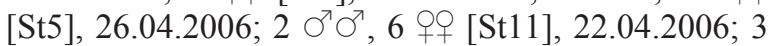
$\sigma^{7}, 11$ 우 [St14], 19.04.2006; 1 ○, 1 ㅇ [StGr7],

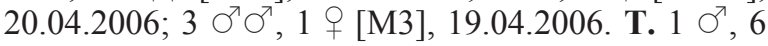


우 [St2], 21.04.2012; 3 O $^{\top} O^{7}, 5$ 우 [M6], 17.04.2012. Y. $1 \mathrm{O}^{7}$ [F13], 19.06.1997.

Troxochrus scabriculus (Westring, 1851)

M. $2 O^{7} \sigma^{7}, 4$ 우 [B3], 20.04.2006.

Walckenaeria alticeps (Denis, 1952)

M. 3 우 [S2], 23.04.2006; T. $1 \mathrm{O}^{7}$ [F5], 17.04. 2012; 2 우 [F23], 20.04.2012.

Walckenaeria antica (Wider, 1834)

K. 1 ㅇ [F12], 22-29.06.1997. 1878)

Walckenaeria atrotibialis (O. Pickard-Cambridge,

K. 1 ㅇ [FE5], 4.05-7.06.2006.

Walckenaeria cucullata (C.L. Koch, 1836)

T. 2 O$^{7} \sigma^{7}$ [F22], 18-21.04.2012. 1875)

Walckenaeria stylifrons (O. Pickard-Cambridge,

T. 1 [M6], 17.04.2012.

Walckenaeria vigilax (Blackwall, 1853)

K. 1 ㅇ [FE1], 4.05-7.06.2006.

Fam. LIOCRANIDAE (2)

Agroeca cuprea Menge, 1873

K. 2 OP [StGr3], 1 [F10], 4.05-7.06.2006; 1 ㅇ [F12] 29.06.1997; 2 우 [FE1], $1 \bigcirc^{7}, 6$ 우 [FE5], 4.057.06.2006. M. 1 ㅇ [M5], 1 ㅇ [W1], 7.05-9.06.2007. T. 1 [ [St2], 17-22.04.2012, ES; 1 \% [StGr7], 19.04.2012, $1 \mathrm{O}^{7}$ [StGr9], 20.04.2012, ES.

Agroeca lusatica (L. Koch, 1875)

K. 2 ㅇ [FE5], 4.05-7.06.2006. M. 1 i [M5], 7.05-9.06.2007; 1 \& [B7], 9.06.2007. T. [St2], 1 , 17-22.04.2012, ES.

Fam. LYCOSIDAE (20)

Alopecosa cursor (Hahn, 1831)

M. 10 O $^{7} O^{7}, 5$ 우 [St13], 3.05-10.06.2006. Y. 2 $\bigcirc^{7} \sigma^{7}, 6$ 우 [StGr6], 4.05-5.06.2007.

Alopecosa farinosa (Herman, 1879)

K. 2 아 [StGr], $50^{7} \sigma^{7}, 2$ 우 [M10], 1 [ [FE1], 1 ㅇ [FE5], 4.05-7.06.2006. M. 1 O $^{7}, 1$ ㅇ [St13], $1 \mathrm{O}^{7}, 5$ 우우 [StGr1], 3.05-10.06.2006; 2 우 [StGr1], 7.05-9. 06.2007; 1 ㅇ [M1], 29.06.1997; T. $49 \sigma^{7} \sigma^{7}, 1$ 우 [St2], 2 $\sigma^{7}$ [StGr9], 1 ○' [F22], 17-21.04.2012, ES. Y. 1 \% [StGr6], 4.05-5.06.2007.

Alopecosa kovblyuki Nadolny et Ponomarev, 2012

K. 1 [FE5], 4.05-7.06.2006. M. 1 연 [M4], (Nadolny et al., 2012), 3.05-10.06.2006; 1 \% [StGr2], 3 우 [M12], 3 우 [W1], 7.05-9.06.2007. Y. 1 † [StGr6], 1 ㅇ [FE1], 4.05-5.06.2007.
Alopecosa pulverulenta (Clerck, 1758)

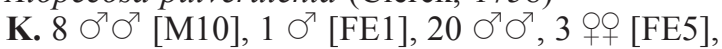
4.05-7.06.2006. M. $24 O^{7} O^{7}, 3$ 우 [StGr1], $2 O^{7} O^{7}, 1$ ㅇ [StGr2], 15 ○ $^{7} \sigma^{7}, 26$ 우 [M4], 8 ○ $^{7} \sigma^{7}, 10$ 우 [M5], 27 $\mathrm{O}^{7} \sigma^{7}, 8$ 우 [M12], 7.05-9.06.2007; $1 \mathrm{O}^{7}$ [W1], 4 O $^{7} \mathrm{O}^{7}$ [FE4], 3.05-10.06.2006; $21 \mathrm{O}^{7} \mathrm{O}^{7}, 12$ 우 [FE4], 7.059.06.2007. T. 6 O $^{7} \sigma^{7}, 1$ + [StGr7], 17-20.04.2012, ES; $2 \sigma^{7} \sigma^{7}$ [M9], 19.04.2012, ES. Y. $1 \sigma^{7}, 1$ [W4], 4 $\mathrm{O}^{\top} \bigcirc^{\top}, 2$ 우 [FE1], 8 ○ $^{\top} \sigma^{\top}, 3$ 우 [FE6], 4.05-5.06.2007.

Alopecosa schmidti (Hahn, 1835)

K. $24 O^{7} O^{7}, 5$ 우 [StGr3], 4.05-7.06.2006. T. 1 O $^{7}$ [St2], 17-20.04.2012 ES. Y. 1 O' [StGr6], 4.055.06.2007.

Alopecosa sulzeri (Pavesi, 1873)

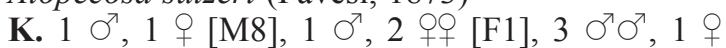
[FE1], 1 o [FE5], 4.05-7.06.2006. Y. $8 \sigma^{7} \sigma^{7}, 1$ ㅇ [F2], 14 O $^{7} \sigma^{\text {T' }}, 6$ Oᄋ [FE2], 4.05-5.06.2007.

Arctosa leopardus (Sundevall, 1833)

Y. 3 O $^{7} \bigcirc^{7}, 5$ 우 [M11], 19.06.1997.

Mustelicosa dimidiata (Thorell, 1875)

K. 5 ○ $^{7} \sigma^{7}$ [F12], 22-29.06.1997.

Pardosa agrestis (Westring, 1861)

K. 2 우 [M10], 22-29.06.1997; 1 क [F10], 1 O $^{7}$ [FE5], 4.05-7.06.2006. M. 1 ㅇ [St13], 9.06.2007; 3 $\sigma^{7} \sigma^{7}$ [M4], 40 O $^{7} \sigma^{7}, 24$ 우 [M5], 15 O $^{\top} \sigma^{7}, 2$ 우 [M12], 7.05-9.06.2007. Y. 1 O $^{7}$ [F2], 4.05.-5.06.2007.

Pardosa alacris (C.L. Koch, 1833)

K. $20 \bigcirc^{7} \sigma^{7}, 2$ Oᄋ $[\mathrm{F} 10], 17 \sigma^{7} \sigma^{7}$ [FE4], 4.057.06.2006. M. $97 \mathrm{O}^{7} \sigma^{7}, 3$ 우 [M12], 7.05-9.06.2007; $237 \mathrm{O}^{\top} \mathrm{O}^{\top}, 12$ 우 [FE5], 1 ㅇ [B7] 3.05-10.06.2006. Y. 17 O $^{7} \sigma^{7}, 9$ 우 [FE6], 4.05-5.06.2007.

Pardosa amentata (Clerck, 1758)

K. 2 O $^{7}, 1$ q [F12], 22-29.06.1997.

Pardosa lugubris (Walckenaer, 1802)

B. 1 + [W3], 19.06.1997. K. 1 i [StGr3], $20^{7} 0^{\top}, 1$

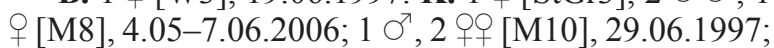
$59 \sigma^{7} \sigma^{7}, 5$ 우 [F1], $7 \sigma^{7} \sigma^{7}$ [F10], $22 \bigcirc^{7} \sigma^{7}, 2$ 아 [FE1], $43 O^{7} \sigma^{7}, 4$ 우 [FE4], 4.05-7.06.2006; 3 O $^{7} \sigma^{7}, 6$ 우 [F12], 29.06.1997; M. 2 O $^{7} \sigma^{7}$ [St13], 2 ○ $^{7} \sigma^{7}$ [StGr1],

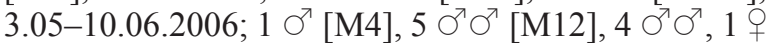
[W1], 7.05-9.06.2007; Y. 1 O$^{7}$ [W4], $8 \sigma^{7} \sigma^{7}, 1$ ㅇ [FE2], 2 O $^{7} \sigma^{7}, 2$ 우 [FE6], 4.05-5.06.2007; 1 O $^{7}, 2$ 우 [F13], 19.06.1997.

Pardosa prativaga (L. Koch, 1870)

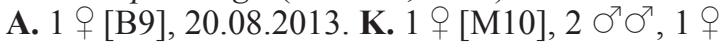
[F12], 22-29.06.1997; M. $60 \mathrm{O}^{7} \mathrm{O}^{7}, 13$ 우 [M12], 7.059.06.2007; $1 \bigcirc^{7}, 6$ 우 [B7], 3.05.2006. Y. 1 + [FE6], 4.05-5.06.2007.

Pirata tenuitarsis Simon, 1876

K. 1 O $^{7}, 1$ ㅇ [M10], 22-29.06.1997. 
Piratula hygrophila (Thorell, 1872) 1997

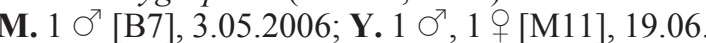

Piratula latitans (Blackwall, 1841)

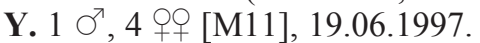

Trochosa robusta (Simon, 1876)

K. 2 O $^{\top}, 1$ q $[\mathrm{StGr} 3], 4.05-7.06 .2006$. M. 1 \% [StGr1], 1 ○', 1 ๆ [M5], 7.05-9.06.2007; 1 ○ [W1], 3.05-10.06.2006. T. $7 \bigcirc^{7} \sigma^{\top}$ [St2], 17-22.04.2012, ES

Trochosa ruricola (De Geer, 1778)

K. 1 \% [FE5], 4.05-7.06.2006. М. 10 ○ $^{\top}, 1$ \% [M12], 7.05-9.06.2007.

Trochosa terricola Thorell, 1856

K. 2 ơ [M1], 29.06.1997; 2 ○ ○', 2 우 [M8],

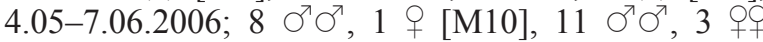

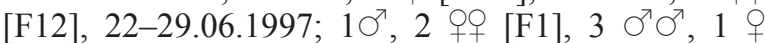
[F10], 2 우 [FE1], 4 ○ $^{\top}, 1$ ㅇ [FE5], 4.05-7.06.2006. M. $4 \sigma^{\top} \sigma^{\top}, 4$ 우 [M4], $8 \sigma^{\top} \sigma^{\top}, 1$ ㅇ [M12], 7.059.06.2007; 2 ○์ ○, 5 Oด [W1], 3.05-10.06.2006; 2 $\sigma^{\top} \sigma^{\top}, 3$ 우 [W1] 7.05-9.06.2007; 1 ○', 1 [FE4], 3.05-10.06.2006. T. $2 \sigma^{7} \sigma^{\top}$ [F22] 18-21.04.2012. Y. 2 우 [W4], 2 ○ $\sigma^{\top}, 3$ 우 [F2], 4.05-5.06.2007; 6 ○ $^{\top}$,

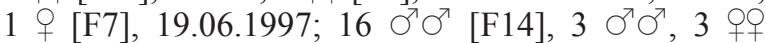
[FE2], 12 O $^{\top}, 9$ 우 [FE6], 4.05-5.06.2007.

Xerolycosa miniata (C.L. Koch, 1834

B. $1+[\mathrm{Gr} 1], 1+[\mathrm{StGr} 5], 19.06 .1997 ;$ K. $1 \sigma^{\top}, 1$ \% [StGr3], 4.05-7.06.2006; $10 \sigma^{7} \sigma^{7}, 13$ oO [M10], 2229.06.1997; 1 ○ [FE1], $2 \sigma^{7} \sigma^{7}, 1$ o [FE5], 4.057.06.2006; M. 2 O $^{\top} \sigma^{7}, 1$ [St13], 3.05-10.06.2006; 3

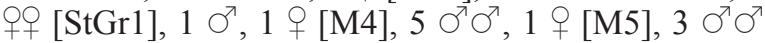
[M12], 7.05-9.06.2007; Y. 14 O$^{\top} \sigma^{\top}, 3$ 우 [StGr6], 1 ㅇ [W3], 1 [FE6], 4.05-5.06.2007.

\section{Fam. MIMETIDAE (2)}

Ero aphana (Walckenaer, 1802)

K. 1 + [M8], 22.08.2013; $2 \sigma^{7} \sigma^{7}$ [FE1], 4.057.06.2006. M. $1+$ [StGr1], 9.06.2007.

Mimetus laevigatus (Keyserling, 1863).

K. $1 \Im^{7}[\mathrm{FE} 1], 3.05-10.06 .2006$. Y. 1 [F13], 19. 06.1997 .

\section{Fam. MITURGIDAE (2)}

Zora pardalis Simon, 1878

M. $1 \sigma^{7}$ [FE5], 3.05-10.06.2006. Y. $2 \sigma^{7} \sigma^{7}$ [FE2], 5 O $^{7}$ [FE6], 4.05-5.06.2007.

Zora spinimana (Sundevall, 1833)

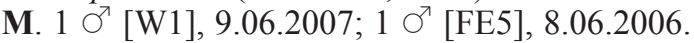

Fam. OXYOPIDAE (2)

Oxyopes heterophthalmus (Latreille, 1804)

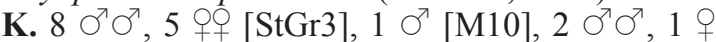
[FE1], 7.06.2006. M. $1 \sigma^{7}$ [St13], $1+$ [M5], 9.06.2007. Y. $1+$ [W4], 1 [ [F2], 5.06.2007.
Oxyopes lineatus Latreille, 1806

A. 2 OO [St1], 20.08.2013. M. 1 [M4], 9.06.2007. Y. 1 [FE6], 5.06.2007.

Fam. PHILODROMIDAE (12)

Philodromus cespitum (Walckenaer, 1802)

K. $1 \sigma^{7}$ [StGr3], 3 of [M8], 7.06.2006; $2 \sigma^{7} \sigma^{7}$

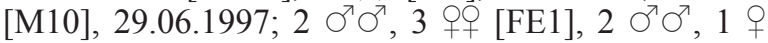

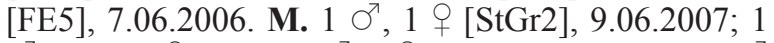
$\sigma^{\top}$ [W1], 1 [FE4], $1 \bigcirc^{7}, 19$ [B7], 7.06.2006. Y. $1 \sigma^{7}$

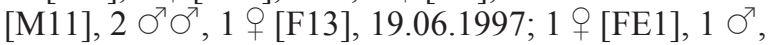
1 ㅇ [FE6], 5.06.2007.

Philodromus dispar Walckenaer, 1826

K. $1 \sigma^{7}, 1$ o [FE1], 7.06.2006. M. 1 [F9],

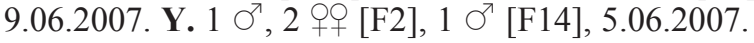

Philodromus marmoratus Kulczyński, 1891

Y. 1 ㅇ [F7], 19.06.1997.

Philodromus rufus Walckenaer, 1826

K. $2 \bigcirc^{7} \bigcirc^{7}, 4$ 우 [FE1], 4 oᄋ [FE5], 1 ㅇ [F10], 7.06.2006; 2 of [F12], 29.06.1997. M. 1 [W1], 1 ㅇ [FE4], 9.06.2007; 1 \% [B7], 7.06.2006. Y. 1 \% [F7], 19.06.1997; 1 ठ [FE6], 5.06.2007.

Rhysodromus fallax (Sundevall, 1883)

M. $1 \sigma^{7}$ [B8], 9.06.2007.

Rhysodromus histrio (Latreille, 1819)

K. 1 $[\mathrm{StGr} 3]$, 7.06.2006. M. 1 [St13], 7.06.2006. Y. 1 ㅇ [StGr6], 5.06.2007.

Thanatus arenarius L. Koch, 1872

K. $4 \sigma^{7} \sigma^{7}, 1$ o $[\mathrm{StGr} 3], 5 \sigma^{7} \sigma^{7}[\mathrm{M} 8], 4.05-7.06$. 2006. M. $5 \sigma^{7} \sigma^{7}$ [St13], $6 \sigma^{7} \sigma^{7}$ [StGr1], 3.05-10.06. 2006; $1 \sigma^{7}, 1$ ㅇ [StGr2], 7.05-9.06.2007; $21 \sigma^{7} \sigma^{7}, 1$ 우 [M4], 39 ठ ठ $\sigma^{7}$ [M5], 1 ○ [M12], 7.05-9.06.2007; 3 $\sigma^{7} \sigma^{7}[\mathrm{~W} 1], 3.05-10.06 .2006$. Y. $25 \sigma^{7} \sigma^{7}$ [StGr6], 3 $\sigma^{7} \sigma^{7}$ [FE1], 1 + [FE6], 4.05-5.06.2007.

Thanatus oblongiusculus (Lucas, 1846)

K. 1 \& [FE1], 6.06.2007.

Thanatus striatus C.L. Koch, 1845

M. 1 \% [FE4], 9.06.2007.

Thanatus vulgaris Simon, 1870

B. $3 \bigcirc^{\top} \bigcirc^{\top}, 4$ q 0 [StGr5], 19.06.1997.

Tibellus macellus Simon, 1875

K. 1 क [M8], 7.06.2006. M. 1 q [St13], 7.06.2006.

Tibellus oblongus (Walckenaer, 1802)

K. 1 [M8], 7.06.2006; 1 [FE5], 7.06.2006. M. $3 \sigma^{7} \sigma^{7}$ [St13], 7.06.2006; 1 q [FE4], 7.06.2006; $2 \sigma^{7} \sigma^{7}$ [FE4], 9.06.2007. T. 1 \% [St2], 20.04.2012, ES.

Fam. PHOLCIDAE (3)

Pholcus opilionoides (Shrank, 1781)

B. $2 \sigma^{7} \sigma^{7}, 7$ OP [Gr1], 19.06.1997. Y. 3 우 [F7], 19.06.1997. 
Pholcus phalangioides (Fuesslin, 1775)

M. $2 \bigcirc^{\top} \sigma^{7}$ [D1], 9.06.2007.

Pholcus ponticus Thorell, 1875 2006.

K. 1 \% [D2], 22.08.2013. M. $2 \bigcirc^{7} \sigma^{7}[\mathrm{D} 1], 7.06$.

\section{Fam. PHRUROLITHIDAE (2)}

Phrurolithus festivus (C.L. Koch, 1835)

B. 2 oq [Gr1], 1 o [W3], 19.06.1997. K. $1 \sigma^{7}$ [M10], 29.06.1997; $2 \sigma^{7} \sigma^{7}[\mathrm{~F} 1], 7.05 .2006 ; 1 \%$ [F1], 1 $\sigma^{\top}, 1$ ㅇ [F10], 7.05.2006; $2 \sigma^{7} \sigma^{7}, 3$ 우 [F12], 2229.06.1997; 1 ㅇ [F12], 29.06.1997; 1 ㅇ [FE5], 4.057.06.2006; 1 ○ , 6.06.2007. М. 1 ऽ, 1 \% [W1], 9.06. 2007; $2 \bigcirc^{7} \sigma^{7}, 1$ [B7], 3.05.2006. Y. $1 \bigcirc^{7}$ [W4], 4.055.06.2007; 1 ○', 2 + + [F14], 5.06.2007.

Phrurolithus pullatus Kulczyński, 1897

K. 1 O' $^{\text {[FE1] }}$, 7.06.2006.

Fam. PISAURIDAE (2)

Pisaura mirabilis (Clerck, 1758)

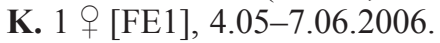

Pisaura novicia (L. Koch, 1878)

K. 4 OQ [M10], 22-29.06.1997; 1 ○, 2 OQ [FE5], 1 $\sigma^{7}$ [F10], 7.06.2006. M. 1 ㅇ [StGr1], 1 ○, 1 क [FE4], 3.05-10.06.2006; 1 ㅇ [B7], 7.06.2006. Y. 2 O$^{\top} \sigma^{\top}, 6$ 우 [StGr6], 4.05-5.06.2007; 4 qo [M11], 19.06.1997; 1 $\sigma^{\top}$ [W3], $1 \bigcirc^{\top}$ [F14], 1 ㅇ [FE2], $1 \bigcirc^{7}$ [FE6], 4.055.06 .2007

\section{Fam. SALTICIDAE (30)}

Aelurillus v-insignitus (Clerck, 1758)

Y. $3 \sigma^{7} \sigma^{7}, 1$ \% [StGr6], $1 \sigma^{7}$ [FE2], 4.05-5.06.2007. T. $1 \sigma^{7}$ [StGr9], 20.04.2012, ES.

Asianellus festivus (C.L. Koch, 1834)

K. $1 O^{7}$ [StGr3], 4.05-7.06.2006. M. $1 \sigma^{7}$ [StGr2],

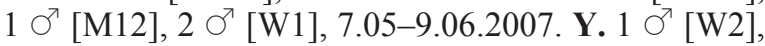
4.05.-5.06.2007.

Ballus chalybeius (Walckenaer, 1802)

K. 3 우 [FE1], 7.06.2006; 1 \& [FE1], 6.06.2007; 1 ㅇ [F1], 2 oㅇ [FE5], 1 \% [F10], 7.06.2006. M. 1 ठ [StGr2], $1 \sigma^{7}$ [M12], 7.05-9.06.2007. T. 1 ○', juv. [StGr9], 20.04.2012, ES. Y. $1 \sigma^{7}$ [W2], 4.05.2007; 1 ㅇ [F14], 5.06.2007.

Carrhotus xanthogramma (Latreille, 1819)

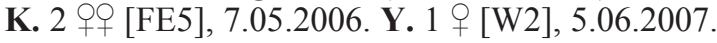

Euophrys frontalis (Walckenaer, 1802)

B. $1 \sigma^{7}$ [W3], 19.06.1997. K. 1 [M8], $1 \sigma^{7}, 3$ \% [FE1], 7.06.2006; 1 O $^{7}$ [FE5], 4.05-7.06.2006. M. 1

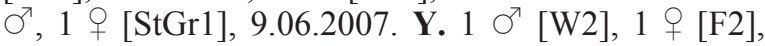
4.05-5.06.2007.
Euophrys petrensis (C.L. Koch, 1837)

K. 1 \& $[\mathrm{StGr} 4]$, 29.06.1997.

Evarcha arcuata (Clerck, 1758)

A. 1 [St1], $1 \sigma^{\top}, 1$ [FE6], 20.08.2013. K. $1 \sigma^{\top}, 1$ q [M8], 6.06.2007; $1 \sigma^{\top}$ [F12], 29.06.1997; 1 [FE1], 7.06.2006; $1 \sigma^{\top}$ [FE1], 5.05.2007; $1 \sigma^{\top}, 1$ ㅇ [FE5], 7.05.2006; 2 우 [FE5], 6.06.2007. М. 1 q [M4], 7.05.2007; 2 ○ $\sigma^{7}$ [M12], 7.05-9.06.2007; 1 [M12], 23.08.2013; 1 क [W1], 1 \% [FE4], 9.06.2007; 1 क [B7], 7.05.2007. Y. 1 ○, 1 \% [W2], 1 \% [FE6], 4.055.06.2007.

Evarcha falcata (Clerck, 1758)

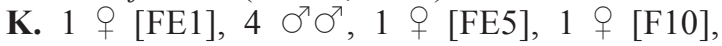
7.06.2006.

Heliophanus auratus C.L. Koch, 1835

K. 3 [M8], 7.06.2006; $1 \sigma^{7}[\mathrm{FE} 1]$, 7.05.2006. M. 2 우 [B7], 7.06.2006.

Heliophanus cupreus (Walckenaer, 1802)

K. $1 \sigma^{\top}, 1$ \& [StGr3], 7.05.2006; $1 \bigcirc^{\top}, 4$ 우 [StGr3], 7.06.2006; $1 \sigma^{7}[\mathrm{M} 10], 2 \sigma^{7} \sigma^{7}$ [F12], 29.06.1997; 1 表 [W1], 7.06.2006; 2 ○ా 2 +

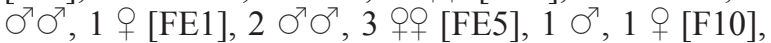

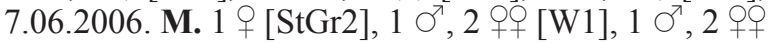
[FE4], 9.06.2007. Y. 1 ठ $^{7}$ [W2], $2 \sigma^{7} \sigma^{7}$ [FE2], 4.055.06.2007; 1 ○, 1 ○ [FE2], 4.05.2007; 1 \% [FE6], 5.06 .2007

Heliophanus flavipes (Hahn, 1832)

A. $3 \sigma^{7} \sigma^{7}$ [FE7], 20.08.2013. K. $2 \sigma^{7} \sigma^{7}$ [StGr3], 7.05.2006, 1 ऽ, 9 우 [StGr3], 7.06.2006; 1 ऊ, 6 우 [M8], 7.06.2006; 1 or, 3 of [W1], 7.06.2006; 1 ठ [FE1], 7.05.2006; 1 ○, 1 क [FE1], 1 ○ [FE5],

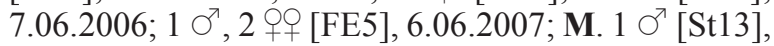
3.05.2006; 1 \% [StGr1], $1 \sigma^{7}$ [M4], 1 \& [M5], 1 \% [W1], $1 \sigma^{7}$ [FE4], 9.06.2007. Y. 1 勇, 1 क [W2], $2 \sigma^{7} \sigma^{7}$ [FE6], 5.06.2007.

Heliophanus lineiventris Simon, 1868

M. 1 [StGr1], 9.06.2007. Y. $1 \Im^{7}, 3$ ㅇ [StGr6], 2 ㅇ [W2], 5.06.2007.

Heliophanus patagiatus Thorell, 1875

B. 1 [ [Gr1], 19.06.1997. K. 5 + [F12], 29.06.1997; $1 \sigma^{7}$ [FE1], 7.05.2006; 1 ㅇ [FE1], 6.06.2007. M. 1 \% [W1], 9.06.2007. Y. $1 \sigma^{7}, 1$ \% [M2], 19.06.1997; $1 \sigma^{7}$ [W2], 1 \% [FE2], 5.06.2007.

Heliophanus simplex Simon, 1826

K. $1 \sigma^{7}$ [StGr3], 7.06.2006; $1 \sigma^{7}$ [FE1], 7.05.2006; $1 \sigma^{7}$ [FE1], 7.06.2006. М. $1 \sigma^{7}$ [W1], 9.06.2007.

Macaroeris flavicomis (Simon, 1884)

K. 2 + [FE1], 7.06.2006. Y. 1 \% [W2], 5.06.2007.

Marpissa muscosa (Clerck, 1758)

Y. 1 \% [F7], 19.06.1997. 
Mendoza canestrinii Ninni, 1868

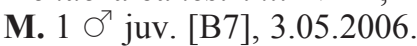

Myrmarachne formicaria (De Geer, 1778)

K. $1 O^{7}$ [M10], 29.06.1997.

Pellenes nigrociliatus (Simon, 1875)

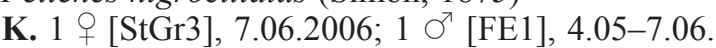
2006.

Pellenes seriatus (Thorell, 1875)

K. 1 \& [StGr3], 7.06.2006; 1 \% [M8], 6.06.2007; 1 o [FE1], 6.06.2007; 1 O $^{7}$ [FE8], 4.05-7.06.2006.

Phlegra fasciata (Hahn, 1826)

M. [M5], 1 ○', 7.05-9.06.2007. Y. 1 ○ [W2], 4. 05-5.06.2007.

Pseudicius encarpatus (Walckenaer, 1802)

K. 1 \&FE1], 6.06.2007. Y. 1 \&W2], 5.06.2007.

Pseudeuophrys obsoleta (Simon, 1868)

B. $1 \sigma^{\top}$ [StGr5], $1 \sigma^{7}, 2$ 우 [W3], 19.06.1997.

Salticus scenicus (Clerck, 1758)

B. $4 \bigcirc^{\top} \sigma^{\top}, 3$ 우 [Gr1], 19.06.1997. K. 4 ㅇ [StGr3], 7.05.2006; 1 + [M8], 6.06.2013; $2 \sigma^{\top} \sigma^{\top}, 2$ o [FE1], 7.05.2006; 1 q [FE1], 7.06.2006; 1 q [FE1], 6.06.2007. M. 1 ㅇ [W1], 9.06.2007.

Sibianor aurocinctus (Ohlert, 1865)

K. 1 q [FE5], 4.05-7.06.2006.

Sitticus dzieduszyckii (L. Koch, 1870)

Y. 1 O’ $^{7}$ 2] ], 19.06.1997.

Sitticus pubescens (Fabricius, 1775)

B. $1 \sigma^{7}$ [StGr5], 19.06.1997. T. $4 \bigcirc^{\top} \sigma^{\top}, 1+$ [StGr8], 18.04.2012, ES.

Sitticus zimmermanni (Simon, 1877)

K. 1 \% $[\mathrm{StGr} 3], 4.05-7.06 .2006 ; 1 \bigcirc^{\top}$ [FE1], 6.06 .2013

Synageles hilarulus (C.L. Koch, 1846)

Y. 1 을 $]$, 4.05.2007.

Synageles subcingulatus Simon, 1878

M. $1 \bigcirc^{\top}$ [StGr1], 2 우 [M5], 9.06.2007.

Fam. TETRAGNATHIDAE (9)

Metellina segmentata (Clerck, 1758)

A. $1 \sigma^{7}, 2$ OQ [FE7], 20.08.2013. K. $2 \sigma^{7} \sigma^{7}$ [FE5],

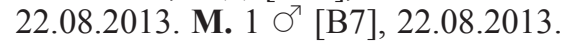

Pachygnatha clercki Sundevall, 1823

K. 3 0 [FE5], 4.05-7.06.2006. М. $4 \bigcirc^{7} \sigma^{7}$ [B7], 7.05.2006.

Pachygnatha degeeri Sundevall, 1830

A. 1 T [B9], 20.08.2013. M. 21 ㅇ [M12], 2 우 [W1], 7.05-9.06.2007. K. 1 [M8], 4.05-7.06.2006;
$1 \%[\mathrm{~F} 1], 1 \sigma^{7}$ [FE1], 07.05.2006. Y. 1 \% [W2], 1 \% [F14], 7 + [FE2], $14 \sigma^{7} \sigma^{7}, 10$ ○ी [FE6], 4.05-5.06.2007.

Pachygnatha listeri Sundevall, 1830

M. 1 ऽ, 1 \% [FE5], 07.05.2007.

Tetragnatha extensa (Linnaeus, 1758)

K. 1 O [M8], 7.06.2006. M. 1 \& [St13], 9.06.2006; 1 † [FE4], 8.06.2006.

Tetragnatha montana Simon, 1874

A. 1 [B9], 20.08.2013. K. $2 \sigma^{7}, 1$ [FE5], 7. 06.2006; 1 क [FE5], 6.06.2007; 3 ○ $\sigma^{7}$ [F9], 7.06.2006;

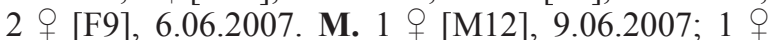
[FE4], 8.06.2006; 1 [FE4], $1 \bigcirc^{7}, 1$ [ [B7], 9.06.2006. Y. 1 OFE6], 5.06.2007.

Tetragnatha nigrita Lendl, 1886

A. $2 \sigma^{\top} \sigma^{\top}, 1+[$ [B9], 20.08.2013. K. 1 \& [FE5], 22.08.2013. М. 1 ठ [W1], 9.06.2007.

Tetragnatha obtusa C.L. Koch, 1837

K. $1 \Im^{7}$ [FE1], 7.06.2006.

Tetragnatha pinicola $\mathrm{L}$. Koch, 1870

K. $1 \sigma^{7}, 1$ \% [FE1], 6.06.2007. M. $1 \sigma^{7}$ [W1], 2 $\sigma^{7} \sigma^{7}$ [FE4], 9.06.2007. Y. 3 Oᄋ [FE2], 1 ○, 3 우 [FE6] 5.06.2007.

Fam. THERIDIIDAE (19)

Asagena phalerata (Panzer, 1801)

K. $1 \sigma^{\top}$ [FE5], 4.05-7.06.2006. M. $1 \sigma^{7}$ [St13], 3.05-10.06.2006; 1 ○ [M4], 2 ○ $^{7}$ [M12], 7.059.06.2007.

Crustulina guttata (Wider, 1834)

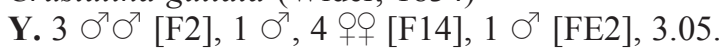
2007.

Cryptachaea riparia (Blackwall, 1854)

K. 1 iFE1], 7.06.2006.

Dipoena melanogaster (C.L. Koch, 1837)

K. $1 \sigma^{7}$ [M1], 1 \% [W2], 29.06.1997; 1 + [FE1] 7.06.2006.

Enoplognatha ovata (Clerck, 1758)

K. 1 ○ [M8], 1 \% [F10], 7.06.2006; 1 \% [F12], 29.06.1997. М. $1 \sigma^{7}$ [FE4], 9.06.2007; 1 フ [B7], 7.06.2006. Y. $1 \bigcirc^{7}$ [FE2], 5.06.2007.

Enoplognatha thoracica (Hahn, 1833)

B. 1 O, 4 of [W3], 19.06.1997. K. 1 \% [FE5], 4.05-7.06.2006. M. 1 ठ [M4], 7.05-9.06.2007. Y. 1 $\sigma^{7}$ [FE2], 1 ○ [FE6] 4.05-5.06.2007.

Episinus truncatus Latreille, 1809

K. $1 \sigma^{7}$ [FE5], 22.08.2013. 
Euryopis quinqueguttata Thorell, 1875

T. 1 \& [StGr7], 19.04.2012, ES.

Heterotheridion nigrovariegatum Simon, 1873

K. 1 ○ [StGr3], 7.06.2006. Y. 2 우 [FE6], 5. 06.2007 .

Neottiura bimaculata (Linnaeus, 1767)

K. $1 \sigma^{7}[\mathrm{FE} 1], 1$ \& $[\mathrm{F} 10]$, 7.06.2006. Y. $1 \sigma^{7}$ [FE2], 5.06.2007.

Neottiura suaveolens (Simon, 1879)

K. $2 \sigma^{\top} \sigma^{\top}, 3$ 우 [StGr3], 7.06.2006; 6 우 [M1], 29.06.1997; 2 ○ O $^{7}$ [M8], 7.05.2006; 3 우 [M8], 5.06 .2007

Parasteatoda lunata (Clerck, 1758)

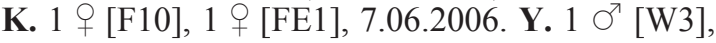
5.06.2007.

Parasteatoda tepidariorum (C.L. Koch, 1841)

M. 1 q [W1], 19.08.2013. Y. 1 q [F7], 19.06.1997.

Phylloneta impressa (L. Koch, 1881)

A. 1 o [St1], 1 o [M11], 20.08.2013. K. $1 \sigma^{7}$

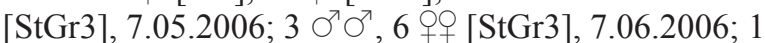
ㅇ [StGr3], 22.08.2013; 1 ○, 4 ơ [M8], 7.06.2006; 2 우 [M8], 22.08.2013; 1 ㅇ [F12], 29.06.1997; 1 ठ, 4 우 [FE1], 7.06.2006; $1 \bigcirc^{\text {T }}$ [FE5], 7.05.2007; 1 ㅇ [FE5], 6.06.2007; 2 우 [FE5], 22.08.2013. М. 1 ऽ, 1 [St13], 3.05.2006; $2 \sigma^{7}, 1$ + [St13], 7.06.2006; 3 $\sigma^{\top} \sigma^{\top}, 1$ ㅇ [StGr2], 1 \% [M4], 9.06.2007; $3 \sigma^{\top} \sigma^{\top}$ [M5], 7.05.2007; 3 ㅇ [M5], 1 ○', 2 우 [W1], 9.06.2007; 2 우 [W1], 19.08.2013. Y. $1 \bigcirc^{\top}$ [StGr6], 4.05.2007.

Platnickina tincta (Walckenaer, 1802)

Y. 1 \% [W4], 5.06.2007.

Simitidion simile (C.L. Koch, 1836)

B. [StGr5] 1 , $19.06 .1997 ;$ K. 4 o [StGr3], 7.05.2006; 1 [M1] 29.06.1997; 1 ○', 6 우 [M8], 7.05.2006; 1 ㅇ [M10], 29.06.1997; 1 O , 3 우 [FE1], 1 $\sigma^{7}$ [FE5] 7.05.2006. М. 1 ○ [W1], 1 \% [FE4], 9.06.2007. Y. 1 O $^{7}, 1$ [FE6], 5.06.2007.

Steatoda albomaculata (De Geer, 1778)

M. $1 \sigma^{7}$ [St13], 7.05.2007.

Theridion innocuum Thorell, 1875

M. 1 \% [StGr1], 7.05-9.06.2007; 1 \% [M4], 9. 06.2007 .

Theridion varians Hahn, 1833

K. $2 \sigma^{\top} \sigma^{\top}, 5$ 우 [FE1], 2 우 [F10], 7.06.2006. M. 1 $\sigma^{7}, 1$ [FE4], 8.06.2006; 1 [ [FE4], 9.06.2007. Y. $1 \sigma^{7}$ [FE2], $1 \bigcirc^{\top}$ [FE6], 5.06.2007.

\section{Fam. THOMISIDAE (23)}

Ebrechtella tricuspidata (Fabricius, 1775)

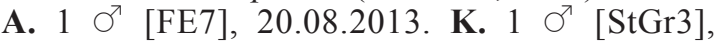
7.06.2006; 1 + [M8], 7.05.2006; 1 क [W2], 29.06.1997;

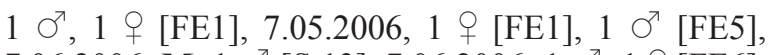
7.06.2006. M. $1 \sigma^{\top}$ [St13], 7.06.2006; $1 \bigcirc^{\top}, 1$ [FE6], 1 을 [ 3.05.2007. Y. $1+[\mathrm{F} 14], 1+$ [FE2], 5.06.2007.

Misumena vatia (Clerck, 1758)

A. 1 [ [FE7], 20.08.2013. K. $1 \sigma^{r}, 1+$ [StGr3], 7.06.2006; $1 \sigma^{\top}$ [M8], 7.05.2006; 1 ㅇ [FE1], 1 ○ , 1 [FE5], 7.06.2006; M. $1 \sigma^{\top}$ [W1], 9.06.2007; 1 \% [FE4], 7.06.2006.

Ozyptila atomaria (Fabricius, 1775)

T. 2 우 [StGr7], 19-20.04.2012, ES; 1 + [StGr9], 20.04.2012, ES. Y. 1 \% [FE2], 4.05-5.06.2007.

Ozyptila claveata (Walckenaer, 1937)

K. $1 \bigcirc^{7}, 4$ O० [M8], 4.05-7.06.2006; 7 ○ $^{7}$ [M10], 22-29.06.1997; $1 \sigma^{\top}, 1$ ㅇ [F1], $3 \sigma^{7} \sigma^{7}, 1$ ㅇ [FE1], $1 \sigma^{7}$ [FE5], 4.05-7.06.2006. M. $1 \sigma^{7}, 1$ क [StGr1], 3.0510.06.2006; $3 \sigma^{7} \sigma^{7}, 2$ 우 [W1], 7.05-9.06.2007. Y. 3

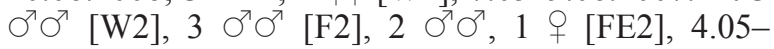
5.06 .2007 .

Ozyptila praticola (C.L. Koch, 1837)

K. $11 \sigma^{7} \sigma^{7}[\mathrm{M} 10], 7 \sigma^{7} \sigma^{7}, 1+$ [F12], 22-29.06.1997; $1 \sigma^{7}$ [FE5], 4.05-7.06.2006; M. $2 \sigma^{7} \sigma^{7}$ [M12], 7.059.06.2007. Y. 1 O $^{7}$ [W2], 4.05-5.06.2007; [F7], $4 \sigma^{7} \sigma^{7}$, 19.06.1997; 3 ○ ○' [F14], 4.05-5.06.2007.

Ozyptila scabricula (Westring, 1851)

K. $1 \sigma^{7}$ [FE5], 4.05-7.06.2006. M. $1 \sigma^{7}$ [W1], 3.05-10.06.2006. T. 7 ○ $\sigma^{7}$ [St2], 17-22.04.2012, ES; $1 \sigma^{7}$ [StGr9], 20.04.2012 ES. Y. $1 \sigma^{7}$ [W2], 4.055.06.2007.

Ozyptila simplex (O. Pickard-Cambridge, 1862)

Y. 1 \& [F7], 19.06.1997.

Pistius truncatus (Pallas, 1772)

K. $1 \sigma^{7}$ [FE1], 7.05.2006.

Runcinia grammica (C.L. Koch, 1837)

A. 2 oq juv. [FE7], 20.08.2013; K. $2 \bigcirc^{7} \sigma^{7}, 6$ 우 [M1], 29.06.1997; Y. 1 ○ [W2], 2 o+ juv. [FE6], 5.06.2007.

Synaema aff. globosum (Fabricius, 1775)

A. 2 우 juv. [B9], 20.08.2013.

Thomisus onustus Walckenaer, 1805

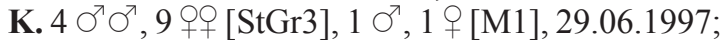
1 ○ [M8], 1 \% [FE1], 7.06.2006. M. 3 of [St13], 9.06.2007.

Tmarus piger (Walckenaer, 1802)

Y. $1 \sigma^{7}$ [FE1], 3.05.2007

Xysticus acerbus Thorell, 1872

K. $1 \sigma^{7}$ [StGr3], 7.06.2006; 1 [M1], 29.06.1997; 19 [FE5], 7.05.2006. M. $1 \sigma^{7}$ [FE4], 9.06.2006. T. 2 $\mathrm{O}^{\top} \mathrm{O}^{\top}$ [St2], 17-22.04.2012 ES; 1 [ [StGr7], 22.04.2012, ES; $1 \sigma^{\top}$ [StGr9], 17.04.201, ES. 
Xysticus cristatus (Clerck, 1758)

K. $3 \bigcirc^{7} \bigcirc^{7}, 3$ oᄋ [StGr3], 7.05.2006; 11 우 [StGr3], 7.06.2006; 2 우 [M8], 7.05.2006; $2 \sigma^{7} \sigma^{7}, 3$ 우 [M8], 7.06.2006; 1 ○', 4 우 [M8], 6.06.2007; $3 \sigma^{7} \sigma^{7}, 10$ 우 [FE1], 7.05.2006, 1 \% , 6.06.2006; 1 [FE1], 6.06.2007; $2 \sigma^{7} \sigma^{7}$ [FE5], 7.05.2006; 1 q [FE5], 7.06.2006; 3 \%o [FE5], 6.06.2007. M. $14 \sigma^{7} \sigma^{7}, 17$ of [St13], 3.05.2006; 1 [St13], 7.06.2006; $1 \sigma^{\top}, 1$ q [StGr2], 2 ○ $\sigma^{7}$ [M4], 5 O$^{7}, 3$ 우 [M5], 1 ○ [M12], 7.05-9.06.2007; 3 $\sigma^{7} \sigma^{7}, 1$ q [W1], 9.06.2007; 2 ○ $\sigma^{7}$ [FE4], 3.05.2006. T. $2 \sigma^{7} \sigma^{7}, 1$ [St2], 17-22.04.2012, ES. Y. $1 \sigma^{7}$ [StGr6], 4.05-5.06.2007, $2 \sigma^{\top} \sigma^{7}, 3$ 우 [StGr6], 5.06.

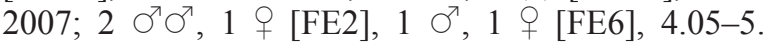
06.2007 .

Xysticus kochi Thorell, 1872

K. $3 \sigma^{7} \sigma^{7}$ [StGr3], 7.05.2006; 2 of [StGr3], 7.06.2006; 1 + [M1], 29.06.1997; 3 ○', 2 우 [M8], 7.05.2006; 1 ㅇ [M10], 2 우 [F12], 22-29.06.1997; 2 ơ $\sigma^{\top}, 2$ 우 [FE1], 7.05.2006; 1 ○ [FE5], 4.057.06.2006; 1 ठ, 1 \% [F10], 7.06.2006. M. $7 \sigma^{7} \sigma^{7}, 1$ O [St13], 3.05-10.06.2006; 1 ㅇ [St13], 7.06.2006; 1 య,

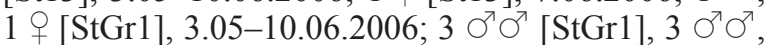
$2+$ [StGr2], 10 O $^{7}, 1$ \% [M5], 1 O $^{7}$ [M12], 7.059.06.2007; $1 \sigma^{7}$ [W1], 3.05.2006; 1 क [FE4], 7.06.2006; T. $1 \sigma^{7}$ [St2], 20.04.2012 ES; $1 \bigcirc^{7}$ [StGr7], 18.04.2012

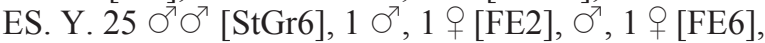
4.05-5.06.2007.

Xysticus laetus Thorell, 1875

K. 1 \& [StGr3], 7.05.2006. M. $2 \sigma^{7} \sigma^{7}$ [M3], 21.04.2012 ES. T. $1 \sigma^{7}$ [StGr7], 19.04.2012 ES.

Xysticus lanio C.L. Koch, 1835

M. 1 ○ $\left.^{7 \mathrm{M} 8}\right]$, 7.05-9.06.2007.

Xysticus luctator L. Koch, 1870

K. $1 \sigma^{7}$ [M10], 1 ○, 1 ㅇ [F12], 22-29.06.1997; 1 $\sigma^{7}$ [FE5], 4.05-7.06.2006. М. $7 \bigcirc^{7} \sigma^{7}$ [W1], 3.0510.06.2006.

Xysticus luctuosus (Blackwall, 1836)

B. 1 \& StGr5], 19.06.1997.

Xysticus ninnii Thorell, 1872

M. $1 O^{7}$ [StGr2], 9.06.2007.

Xysticus robustus (Hahn, 1832)

K. $1 O^{7}$ [F12], 22-29.06.1997 KE.

Xysticus striatipes L. Koch, 1870

A. $3 \sigma^{7}$ [St1], $4 \sigma^{7} \sigma^{7}$ [FE7], 20.08.2013. K. $3 \sigma^{7} \sigma^{7}$ [StGr3], 22.08.2013; 1 q [M8], 7.06.2006; 4 ○ $^{7}, 1$ \% [M8], 22.08.2013; 1 [FE1], 5.05.2007; M. $2 \sigma^{7} \sigma^{7}$ [StGr1], $1 \sigma^{7}$ [M12], $4 \sigma^{7} \sigma^{7}[\mathrm{~W} 1], 19.08 .2013$. T. 1 ㅇ [StGr7], 19.04.2012 ES. Y. 2 우 [StGr6], 4.055.06.2007.

Xysticus ulmi (Hahn, 1831)

M. $3 \sigma^{7} \sigma^{7}[\mathrm{M} 12], 7.05 .2007 ; 1 \sigma^{7}$ [FE4], 3.05.2006.

\section{Fam. TITANOECIDAE (1)}

Titanoeca schineri L. Koch, 1872

B. $1+[\mathrm{StGr} 5], 2 \sigma^{7} \sigma^{7}, 1+[\mathrm{W} 3], 19.06 .1997$. K. 1

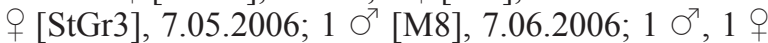
[M10], 3 O+ [F12], 29.06.1997; $2 \sigma^{7} \sigma^{\top}$ [FE1] 6.06.2007. M. 1 [St13], 3.05.2007; $1 \sigma^{7}$ [M4], 7.05-9.06.2007. T. $1 \sigma^{7}, 1 q$ [StGr7]. 19.04.2012 ES. Y. $2 \sigma^{7} \sigma^{7}, 1$ q [M2], 19.06.1997; $1 \sigma^{\top}$ [F2], $1 \sigma^{\top}$ [FE2], $3 \sigma^{\top} \sigma^{\top}$ [FE6], 4.05-5.06.2007.

\section{Fam. ULOBORIDAE (1)}

Uloborus walckenaerius Latreille, 1806

K. $3 \bigcirc^{7} \Im^{7}, 4$ Oᄋ [StGr3], 6.06.2007; 1 \% [M9], $22.08 .2013 ; 3$ 우 [FE1], 7.06.2006; 2 우 [FE1], 6.06.2007. M. 1 \& [St13], 7.06.2006; $1 \sigma^{x}, 1$ [ [StGr2], 7.05-9.06.2007. Y. 1 \% [M2], 19.06.1997; 1 \& [FE2], 3

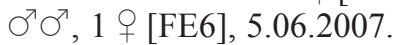

\section{Fam. ZODARIIDAE (1)}

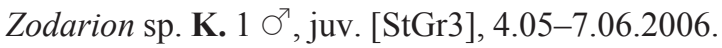

\section{Discussion}

A total of 266 spider species of 28 families has been recorded from the National Nature Park 'Buzkyi Hard' and its vicinity. Of them, the family Linyphidae is most species-rich (61 species). The next species-rich families are ranked as follows: Salticidae, Gnaphosidae, Thomisidae, Araneidae, Lycosidae, Theridiidae, and Philodromidae (Table 2).

The studied local spider fauna appears to be less diverse than those of other well-studied protected areas (Table 2). Although these parks and reserves have different territories and different ratios of steppe, forest and wetland habitats, everywhere the Linyphiidae is a dominant family, Gnaphosidae and Salticidae follow up; only in the 'Sviati Hory', the Salticidae is less diverse than Araneidae, Lycosidae or Theridiidae. In the 'Buzkyi Hard', the species richness of Araneidae, Linyphiidae, Philodromidae, Salticidae and Thomisidae was not much lower than the average in other parks and reserves, while the families Lycosidae, Theridiidae and especially Gnaphosidae were much poorer.

Both combining and separation methods of the faunas comparison have produced similar results (Fig. 2). The three most closely located reserves within the forbbanchgrass steppe subzone (Ukrainian Steppe, Luhanskyi and Razdorskyi) are grouped together at the DCA ordination plot and form a central claster in the dendrogram. They represent vast steppe sites and various plots with arboreal vegetation. The 'Sviati Hory' Park stands apart, as it has a very small steppe area and is known as an "island of the forest-steppe in the steppe zone". The Black Sea Reserve is situated in the south of the bunchgrass steppe subzone. Its high variety of habitats on sandy soils includes saline marshes and marine bay shores that are absent in other reserves. The 
Table 2. Species richness of spider families in nature parks and reserves of the steppe zone of the East European Plain. BH - National Nature Park 'Buzkyi Hard', BSSBR - Black Sea State Biosphere Reserve, USNR — Ukrainian Steppe Nature Reserve, LNR — Luhansk Natural Reserve, SH — National Nature Park 'Sviati Hory', RMR — Razdorsky Museum-Reserve. Species number (\%).

Табл. 2. Видовое богатство семейств пауков в природных парках и заповедниках степной зоны Восточноевропейской равнины. ВH - Национальный природный парк «Бугский Гард», BSSBR — Черноморский государственный биосферный заповедник, USNR - Украинский степной заповедник, LNR - Луганский природный заповедник, SH — Национальный природный парк «Святые Горы», RMR — Раздорский музейзаповедник. Число видов (\%).

\begin{tabular}{|c|c|c|c|c|c|c|}
\hline \multirow{2}{*}{ Family } & \multicolumn{6}{|c|}{ Nature Parks and Reserves } \\
\hline & $\mathrm{BH}$ & BSSBR & USNR & LNR & $\mathrm{SH}$ & RMR \\
\hline Agelenidae & $4(1.5)$ & $3(1.0)$ & $4(1.4)$ & $4(1.2)$ & $4(1.4)$ & $5(1.5)$ \\
\hline Anyphaenidae & $1(0.4)$ & $1(0.3)$ & - & $1(0.3)$ & $1(0.3)$ & $1(0.3)$ \\
\hline Araneidae & $22(8.3)$ & $22(7.3)$ & $23(8.2)$ & $28(8.3)$ & $22(7.6)$ & $19(5.8)$ \\
\hline Atypidae & $1(0.4)$ & $1(0.3)$ & $1(0.4)$ & $1(0.3)$ & $2(0.7)$ & $1(0.3)$ \\
\hline Argyronetidae & - & $1(0.3)$ & $1(0.4)$ & - & $1(0.3)$ & - \\
\hline Clubionidae & $5(1.9)$ & $5(1.7)$ & $8(2.8)$ & $11(3.3)$ & $9(3.1)$ & $8(2.4)$ \\
\hline Dictyniae & $6(2.3)$ & $7(2.3)$ & $9(3.2)$ & $6(1.8)$ & $6(2.1)$ & $7(2.1)$ \\
\hline Dysderidae & $2(0.8)$ & - & $3(1.1)$ & - & $1(0.3)$ & $1(0.3)$ \\
\hline Eresidae & $1(0.4)$ & $1(0.3)$ & $1(0.4)$ & $1(0.3)$ & $1(0.3)$ & $1(0.3)$ \\
\hline Eutrichuridae & $5(1.9)$ & $6(2.0)$ & $5(1.8)$ & $5(1.5)$ & $4(1.4)$ & $7(2.1)$ \\
\hline Gnaphosidae & $26(9.8)$ & $41(13.5)$ & $37(13.1)$ & $38(11.3)$ & $30(10.4)$ & $49(14.8)$ \\
\hline Hahniidae & $1(0.4)$ & $1(0.3)$ & $2(0.7)$ & $2(0.6)$ & $2(0.7)$ & $2(0.6)$ \\
\hline Linyphiiae & $61(22.9)$ & $64(21.1)$ & $64(19.5)$ & $71(21.1)$ & $63(21.9)$ & $57(17.3)$ \\
\hline Liocranidae & $2(0.8)$ & $4(1.3)$ & $2(0.7)$ & $4(1.2)$ & $5(1.7)$ & $6(1.8)$ \\
\hline Lycosidae & $20(7.5)$ & $30(9.9)$ & $22(7.8)$ & $27(8.0)$ & $26(9.0)$ & $27(8.2)$ \\
\hline Mimetidae & $2(0.8)$ & $2(0.7)$ & $2(0.7)$ & $1(0.3)$ & $2(0.7)$ & $4(1.2)$ \\
\hline Miturgidae & $2(0.8)$ & $4(1.3)$ & $2(0.7)$ & $3(0.9)$ & $3(1.0)$ & $4(1.2)$ \\
\hline Oxyopidae & $2(0.8)$ & $2(0.7)$ & $2(0.7)$ & $2(0.6)$ & $1(0.3)$ & $1(0.3)$ \\
\hline Philodromidae & $12(4.5)$ & $13(4.3)$ & $11(3.9)$ & $14(4.2)$ & $16(5.6)$ & $11(3.3)$ \\
\hline Pholcidae & $3(1.1)$ & $1(0.3)$ & $2(0.7)$ & $3(0.9)$ & $2(0.7)$ & $3(0.9)$ \\
\hline Phrurolithidae & $2(0.8)$ & $2(0.7)$ & $3(1.1)$ & $2(0.6)$ & $1(0.3)$ & $2(0.6)$ \\
\hline Pisauridae & $2(0.8)$ & $1(0.3)$ & $3(1.1)$ & $2(0.6)$ & $3(1.0)$ & $2(0.6)$ \\
\hline Salticidae & $30(11.3)$ & 35 (11.6) & $33(11.7)$ & $40(11.9)$ & $21(7.3)$ & $41(12.4)$ \\
\hline Scythodidae & - & $1(0.3)$ & $1(0.4)$ & - & - & $1(0.3)$ \\
\hline Sparassidae & - & $1(0.3)$ & $1(0.4)$ & $1(0.3)$ & $1(0.3)$ & $1(0.3)$ \\
\hline Tetragnathidae & $9(3.40$ & $5(1.7)$ & $6(2.1)$ & $10(3.0)$ & $10(3.5)$ & $8(2.4)$ \\
\hline Theridiidae & $19(7.1)$ & $24(7.9)$ & $18(6.4)$ & $28(8.3)$ & $26(9.0)$ & $31(9.4)$ \\
\hline Thomisidae & $23(8.6)$ & $20(6.6)$ & $22(7.8)$ & $26(7.7)$ & $22(7.6)$ & $25(7.6)$ \\
\hline Titanoecidae & $1(0.4)$ & $3(1.0)$ & $2(0.7)$ & $3(0.9)$ & $3(1.0)$ & $3(0.9)$ \\
\hline Uloboridae & $1(0.4)$ & $1(0.3)$ & - & $1(0.3)$ & $1(0.3)$ & $1(0.3)$ \\
\hline Zodariidae & $1(0.4)$ & $1(0.3)$ & $1(0.4)$ & $1(0.3)$ & - & $1(0.3)$ \\
\hline Total & $266(100)$ & $303(100)$ & $282(100)$ & $336(100)$ & $289(100)$ & $330(100)$ \\
\hline
\end{tabular}




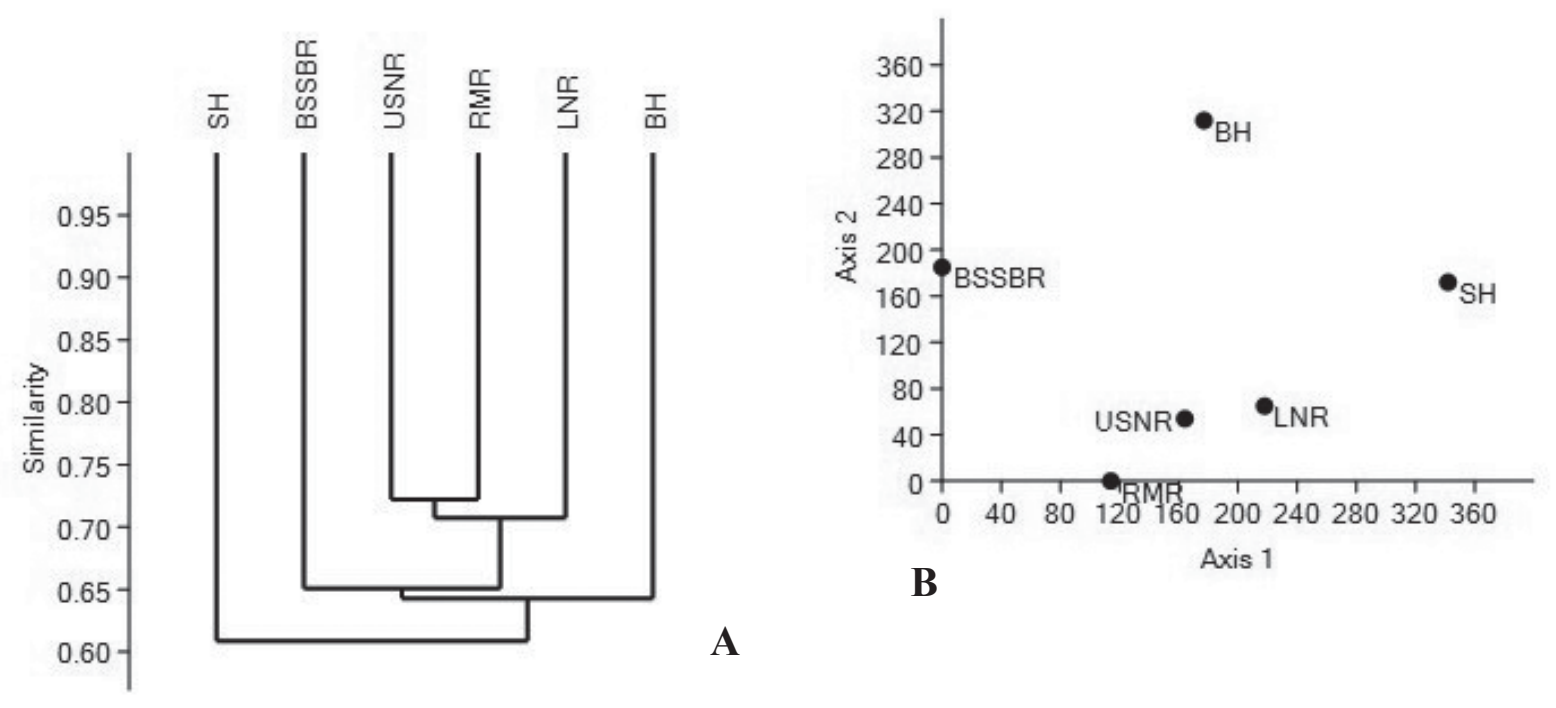

Fig. 2. Comparative analysis of the spider faunas of six conservation areas. A - cluster analysis based on the UPGMA method as a cluster algorithm and the Bray-Curtis index as a similarity measure. B - DCA ordination. For abbreviations, see Table 2.

Рис. 2. Сравнительный анализ фаун пауков шести охраняемых территорий. A - кластерный анализ на основе метода UPGMA как кластерного алгоритма и идекса Брэя-Куртиса как меры сходства. В — DCA ординация. Сокращения см. табл. 2.

'Buzkyi Hard' remote position at the DCA ordination and its least similarity with the steppe reserves can be explained by its westernmost geographical location.

The 'Buzkyi Hard' is inhabited by a number of rare species. For Neottiura suaveolens, it is the second record from Ukraine. Hitherto, N. suaveolens was found in the northeast part of Luhansk Area [Polchaninova, 1990]. In 2008, that area was affected by extensive fire, and since that time $N$. suaveolens has not been registered from there [Polchaninova, 2015b]. Therefore, now the 'Buzkyi Hard' is the only verified locality of this species in Ukraine.

The geographic range of Cyclosa sierre extends from Portugal to Azerbaijan and from south Ukraine to Israel [Kovblyuk, Kastrygina, 2015]. To date, the 'Buzkyi Hard' represents the northernmost known locality of this species. For Canariphantes nanus and Dysdera lata, the Park is their northernmost findings in Ukraine. The Park also lies at the northernmost boundary of the Mimetus laevigatus range (a more detailed information is available in Kovblyuk, Kastrygina, 2015).

For Zelotes apricorum, the Park seems to represent the southern boundary of its range in the East European Plain. Being widely distributed in the forest zone, this species has been found in two localities in the steppe zone only: viz., the National Parks 'Sviati Hory' [Polchaninova, Prokopenko, 2013] and 'Buzkyi Hard' (present data). Both Parks are situated in the uplands with chalky or granitic hills. For three species, Haplodrassus pseudosignifer [Kovblyuk, Kastrygina, 2015], Alopecosa kovblyukii [Nadolny et al., 2012] and Agyneta saaristoi, the 'Buzkyi Hard' is the westernmost known locality of their ranges.

Spiders have long been known as particularly sensitive to habitat disturbances. Their high abundance, wide distribution and vulnerability make spiders ideal candidates for conservation studies [Cardoso, Morano, 2010]. Our investigations have confirmed a high conservation value of the National Nature Park 'Buzkyi Hard'. Besides the typical representatives of steppe and forest habitats, its fauna includes a number of rare species (see above). Some of them seem have boundaries of their geographic ranges that are situated in the Park territory. The next stage of arachnological researches in the Park should be focused on a comparative analysis of spider assemblages and an estimation of the number of rare species.

\section{References}

Adler P.B., Lauenroth W.K. 2003. The power of time: spatiotemporal scaling of species diversity // Ecological Letters. Vol.6. P.749-756.

Barbarych A.I. (chief ed.). 1997. [Geobotanical Regioning of the UkrSSR]. Kyiv: Naukova Dumka. 304 p. [in Ukrainian]

Cardoso P., Morano E. 2010. Te Iberian Spider checklist // Zootaxa. No.2495. P.1-50.

Hammer Ø., Harper D.A.T., Ryan P.D., 2001: PAST: Paleontological Statistics Software Package for Education and Data Analysis. Palaeontology Electronic 4, 9 pp. http://folk.uio.no/ohammer/past

Kovblyuk M.M., Kastrygina Z.A. 2015. [Updated catalogue of the spiders (Arachnida, Aranei) of the Crimea] // Ukrainska Entomofaunistyka. Vol.6. No.2. P.1-81 [in Russian with English summary].

Kovblyuk M.M., Kastrygina Z.A., Omelko M.M. 2012. A review of the spider genus Haplodrassus Chamberlin, 1922 in Crimea (Ukraine) and adjacent areas (Araneae, Gnaphosidae) // ZooKeys. Vol.205. P.59-89.

Nadolny A.A., Ponomarev A.V., Dvadenko K.V. 2012. A new wolf spider species in the genus Alopecosa Simon, 1885 (Araneae: Lycosidae) from Eastern Europe // Zootaxa. No.3484. P.8388 .

Polchaninova N.Yu. 1990. [Comparative characteristics of the spider fauna of the steppes of Left-bank Ukraine] // I.A. Akimov 
(ed.). Novosti faunistiki i sistematiki. Kiev: In-t zool. AN UkrSSR. P.163-167. [in Russian, with English summary].

Polchaninova N.Yu. 2012. [A checklist of spiders (Araneae) of the Chornomorskyi Biosphere Reserve (Ukraine)] // Pryrodnychyi almanakh. Biolohichni nauky. Kherson: PAT 'Khersonska miska drukarnia'. Vyp.18. P.85-108 [in Russian, with English summary].

Polchaninova N.Yu. 2015a. [State of knowledge of the araneofauna of Mykolaiv Region and first data on the spiders of the NPP 'Buzkyi Hard'] // 'Rehionalni aspecty floristychnykh i faunistychnykh doslidjen'. Materialy Druhoi nauchno-praktychnoi konferentsii. Chernivtsi, Druk Art. P.256-258 [in Ukrainian].

Polchaninova N. 2015b. Recovery of spider communities after a spontaneous summer fire in the forb-bunchgrass steppe of eastern Ukraine // Hacquetia. Vol.14. No.1. P.79-96.
Polchaninova N.Yu., Prokopenko E.V. 2013. Catalogue of the spiders (Arachnida, Aranei) of Left-Bank Ukraine. Arthropoda Selecta. Supplement No.2. Moscow: KMK Scientific Press. $268 \mathrm{p}$.

Polchaninova N.Yu., Prokopenko E.V. 2017. Catalogue of the spiders (Arachnida, Aranei) of Left-Bank Ukraine. Addendum 1. 2013-2016. Arthropoda Selecta. Supplement No.4. Moscow: KMK Scientific Press. 115 p.

Ponomarev V.A., Tsvetkova Yu. A. 2003. [Spiders (Aranei) of the Rozdorsky Museum-Reserve territory] // A.V. Ponomarev (ed.). Istoriko-Kulturnye i prirodnye issledovania na territorii Rozdorskogo etnograficheskogo muzeya-zapovednika. Rostov-on-Don: Rostov University. P.167-208. [in Russian].

World Spider Catalogue 2017. World Spider Catalog. Natural History Museum Bern, online at http://wsc.nmbe.ch, version 17.5

Responsible editor D.V. Logunov 